\title{
New Insights and Methods for Recording and Imaging Spontaneous Spreading Depolarizations and Seizure-Like Events in Mouse Hippocampal Slices
}

\author{
Yi-Ling Lu ${ }^{1}$ and Helen E. Scharfman 1,2,3,4* \\ ${ }^{1}$ Center for Dementia Research, The Nathan Kline Institute for Psychiatric Research, Orangeburg, NY, United States, \\ ${ }^{2}$ Department of Child and Adolescent Psychiatry, New York University Langone Health, New York, NY, United States, \\ ${ }^{3}$ Department of Neuroscience and Physiology, New York University Langone Health, New York, NY, United States, \\ ${ }^{4}$ Department of Psychiatry, New York University Langone Health, New York, NY, United States
}

\section{OPEN ACCESS}

Edited by:

Rustem Khazipov,

Institut National de la Santé et de la Recherche Médicale (INSERM),

France

Reviewed by:

R. David Andrew,

Queen's University, Canada

Eszter Farkas,

University of Szeged, Hungary

*Correspondence:

Helen E. Scharfman

Helen.Scharfman@NKI.rfmh.org

Specialty section:

This article was submitted to

Cellular Neuropathology,

a section of the journal

Frontiers in Cellular Neuroscience

Received: 19 August 2021

Accepted: 04 October 2021

Published: 26 November 2021

Citation:

LU Y-L and Scharfman HE (2021)

New Insights and Methods

for Recording and Imaging

Spontaneous Spreading Depolarizations and Seizure-Like

Events in Mouse Hippocampal Slices.

Front. Cell. Neurosci. 15:761423.

doi: 10.3389/fncel.2021.761423
Spreading depolarization (SD) is a sudden, large, and synchronous depolarization of principal cells which also involves interneurons and astrocytes. It is followed by depression of neuronal activity, and it slowly propagates across brain regions like cortex or hippocampus. SD is considered to be mechanistically relevant to migraine, epilepsy, and traumatic brain injury (TBI), but there are many questions about its basic neurophysiology and spread. Research into SD in hippocampus using slices is often used to gain insight and SD is usually triggered by a focal stimulus with or without an altered extracellular buffer. Here, we optimize an in vitro experimental model allowing us to record SD without focal stimulation, which we call spontaneous. This method uses only an altered extracellular buffer containing $0 \mathrm{mM} \mathrm{Mg}^{2+}$ and $5 \mathrm{mM} \mathrm{K}^{+}$and makes it possible for simultaneous patch and extracellular recording in a submerged chamber plus intrinsic optical imaging in slices of either sex. We also add methods for quantification and show the quantified optical signal is much more complex than imaging alone would suggest. In brief, acute hippocampal slices were prepared with a chamber holding a submerged slice but with flow of artificial cerebrospinal fluid (aCSF) above and below, which we call interface-like. As soon as slices were placed in the chamber, aCSF with $0 \mathrm{Mg}^{2+} / 5 \mathrm{~K}^{+}$was used. Most mouse slices developed SD and did so in the first hour of $0 \mathrm{Mg}^{2+} / 5 \mathrm{~K}^{+}$aCSF exposure. In addition, prolonged bursts we call seizure-like events (SLEs) occurred, and the interactions between SD and SLEs suggest potentially important relationships. Differences between rats and mice in different chambers are described. Regarding optical imaging, SD originated in CA3 and the pattern of spread to CA1 and the dentate gyrus was similar in some ways to prior studies but also showed interesting differences. In summary, the methods are easy to use, provide new opportunities to study SD, new insights, and are inexpensive. They support previous suggestions that SD is diverse, and also suggest that participation by the dentate gyrus merits greater attention.

Keywords: spreading depression, intrinsic optical signal, seizures, epilepsy, experimental model, migraine, traumatic brain injury, dentate gyrus 


\section{INTRODUCTION}

Increased neuronal excitability is a common feature underlying several neurological disorders, including but not limited to migraine, epilepsy, traumatic brain injury (TBI), and stroke (Eikermann-Haerter et al., 2013; Noseda and Burstein, 2013; Rogawski, 2013; Kim et al., 2014; Bugay et al., 2020). Although seizures may be the best-known example that results from increased neuronal excitability, spreading depolarization (SD) is another example.

Spreading depolarization is a group of cells that depolarize dramatically and typically begin to fire numerous action potentials (APs). Ultimately firing ceases, neurons appear to lose normal intrinsic electrophysiological properties such as input resistance, and there is a loss of the normal ion gradients across the membrane (Czéh et al., 1993; Somjen, 2001; Hartings et al., 2017). The activity of the subset of neurons is almost synchronous so when electrical activity is monitored extracellularly, the initial depolarization is associated with a significant negative potential shift in an extracellular recording [direct current (DC) recording]. At the peak of the depolarization, action potential firing stops and neurons are silent. This period is associated with a suppression in the extracellular recording and is followed by a slow recovery. During the recovery, glia and ion pumps restore normal ionic gradients (Lian and Stringer, 2004; Dreier, 2011; Varga et al., 2020).

Leão (1944) first reported SD after focal electrical stimulation and its slow spread across the cortex of the anesthetized rabbit. He coined the term "spreading depression (SD)." However, it should be noted that SD sometimes involves a phase of depression that precedes, rather than follows, depolarization (Dreier, 2011; Ayata and Lauritzen, 2015).

There is extensive influx of sodium and calcium, and large efflux of potassium that leads to the loss of ion gradients (Somjen, 2001; Dreier, 2011; Ayata and Lauritzen, 2015). The inflow of cations brings water inside the neuron, which leads to swelling and alters the light transmission through tissue (Dreier, 2011). Therefore, the change in light transmission has been used as a method to monitor SD-associated swelling and the propagation of SD from one brain area to the next (e.g., Obeidat and Andrew, 1998; Anderson and Andrew, 2002; Buchheim et al., 2002).

To gain a better understanding of mechanisms underlying SD, past hippocampal studies have typically used slice models and rats. Many of these studies were conducted in an interface chamber (e.g., Snow et al., 1983; Scharfman, 1997; Buchheim et al., 2002; Reyes-Garcia et al., 2018). In an interface chamber, brain slices lie on a mesh at the interface between artificial cerebrospinal fluid (aCSF) and air. Warm, humidified $\mathrm{O}_{2}$ or $95 \%$ $\mathrm{O}_{2} / 5 \% \mathrm{CO}_{2}$ is vented over the slices (Schwartzkroin, 1975; Haas et al., 1979; Scharfman et al., 2001). Although interface chambers are widely used to study SD, lack of a powerful microscope limits visualization (but see Case and Broberger, 2013). On the other hand, a submerged chamber that is typically used with a high-resolution microscope allows visualization of neurons. Visualization is an advantage because it facilitates patch clamp recordings and optical imaging.

In both types of recording chambers, SDs are often initiated by electrical stimulation or focal application of concentrated potassium solutions, sometimes with a change in the ionic milieu to increase excitability (e.g., Czéh et al., 1993; Buchheim et al., 2002; Lindquist and Shuttleworth, 2012; Eickhoff et al., 2014; Martens-Mantai et al., 2014). When a different approach is used to increase excitability, such as reducing $\mathrm{Mg}^{2+}$ in aCSF, afferent stimuli is typically needed to elicit SD (Mody et al., 1987). Spontaneous SD is rare and delayed (Mody et al., 1987). In a submerged chamber, SD is not found (Anderson et al., 1986; Lewis et al., 1990; Churn et al., 1991; Kojima et al., 1991). Therefore, methods using a submerged chamber would be beneficial. Moreover, use of a low concentration of $\left[\mathrm{Mg}^{2+}\right]_{\mathrm{o}}$ is advantageous to study SD because it is typically a powerful inhibitor of SD (Santos et al., 2016). Indeed, one reason some past studies have had difficulty in producing SD could be due to a relatively high $\mathrm{Mg}^{2+}$ level in the aCSF, which is often done to preserve slices during the dissection. Finally, focal stimulation may not always be effective in eliciting SD. In the dentate gyrus, for example, afferent stimulation can lead to frequency depression, not facilitation (Sloviter, 1991; Scharfman et al., unpublished observations). If the GC axons are stimulated, CA3 does not exhibit SD under normal conditions (Scharfman, 1997).

Here, we report methods to achieve this goal and new findings. Regarding new methods, we used a submerged chamber with flow above and below the slice, and compared results to a classic submerged chamber with more limited flow. When slices were placed in the recording chamber, we began to use aCSF containing $0 \mathrm{mM} \mathrm{Mg}{ }^{2+}$ and $5 \mathrm{mM} \mathrm{K}^{+}$. We refer to this aCSF as " $0 \mathrm{Mg}^{2+} / 5 \mathrm{~K}^{+}$aCSF." SD was recorded in the majority of slices and we refer to SD that occurred, without a time-locked focal stimulus, as "spontaneous" SD. These spontaneous SDs appeared to be spontaneous because they had an unpredictable delay before SD ultimately occurred. Spontaneous SD is usually rare in slices, rare in adult mouse, and rare in a submerged chamber, providing a potentially useful and new approach to complement others. Our methods allowed us to record spontaneous SDs, bursts, and trains of bursts we call SLEs, which is valuable because these different types of epileptiform activity occur in humans. We added intrinsic optical imaging and developed methods to quantify the imaging results, finding a spatiotemporal complexity the quantification identified that was not evident by observation of video. These methods provided additional insight about SD beyond what is known and provide new analysis opportunities to extend our understanding of the dynamics of cell swelling in SD. In our slices, each subfield showed good viability so we followed SD from CA3 not only to CA1 but also the dentate gyrus. Interestingly, the delay and invasion of $\mathrm{SD}$ in the dentate gyrus was complex. We suggest that these methods and novel observations provide further advances in understanding $\mathrm{SD}$ as well as providing a preparation that may be useful to preclinical drug screening.

\section{MATERIALS AND METHODS}

The experimental procedures were carried out in accordance with the National Institutes of Health guidelines and were 
approved by the Institutional Animal Care and Use Committee in The Nathan Kline Institute. All chemicals were obtained from Millipore-Sigma unless otherwise specified.

\section{Animals and Husbandry}

A total of 10 Sprague-Dawley rats (all males, age 21-45 days) and $26 \mathrm{C} 57 \mathrm{BL} / 6$ mice (14 males and 12 females, age 2036 days) were used in the current study (Table 1). The maximum number of animals housed per cage was three for rats and four for mice. All animals were housed using a 12 h light/dark cycle. Food (Purina 5001 Chow, W. F. Fisher) and water were available ad libitum. Numbers of animals that were used in each experimental condition are listed in the Table 1.

\section{Terminology}

Definitions of the terms described below were defined based on past studies of SD and our data. Extracellularly, SD was defined as an event that consists of a negative DC shift with a slow recovery. In our model, SD almost always began with a series of high frequency bursts and then proceeded to a negative DC shift. Intracellularly, SD began with a large depolarization with superimposed high frequency firing of APs at the onset. Subsequently, firing ceased and there was a slow repolarization.

A SLE was defined by multiple criteria to distinguish it as abnormal and seizure-like. Extracellularly, SLEs were defined as multiple bursts of population spikes superimposed on short-lasting positive waves. In slices, where spontaneous population spikes are not normally present, SLEs were easy to distinguish. SLEs were referred to as seizure-like because they showed more bursts than the 1-2 bursts referred to as an epileptiform discharges. SLEs also were composed of bursts at high frequency $(>1 \mathrm{~Hz})$ and some bursts were prolonged (Borck and Jefferys, 1999). Intracellularly, an SLE corresponded to an initial depolarization with APs at the peak, somewhat like a paroxysmal depolarization shift (PDS; Borck and Jefferys, 1999). The SLEs observed in our preparation resembled events that have been called ictaform or ictal events/activity in the past (Anderson et al., 1986; Avoli et al., 2002).

\section{Slice Preparation and Electrophysiological Recording}

Rats or mice were deeply anesthetized using isoflurane (Patterson Veterinary) and then decapitated. Brains were quickly removed and immersed in ice-cold sucrose-containing aCSF (sucrose aCSF, ingredient in mM: 90 sucrose, $80 \mathrm{NaCl}, 2.5 \mathrm{KCl}, 1.25$ $\mathrm{NaH}_{2} \mathrm{PO}_{4}, 25 \mathrm{NaHCO}_{3}, 10$ D-glucose, $4.5 \mathrm{MgSO}_{4}$, and 0.5 $\left.\mathrm{CaCl}_{2}, \mathrm{pH}=7.3-7.4\right)$. Horizontal hippocampal slices $(350 \mu \mathrm{m}$ thick) were obtained using an oscillating tissue slicer (Microm, HM650V, Thermo Fisher Scientific, or VT1200 S, Leica). Brain slices were then transferred to a holding chamber (made inhouse) containing sucrose aCSF. This chamber allowed slices to sit on a mesh several inches from the base of the chamber but still below the surface of aCSF, and the aCSF circulated around the slices. The holding chamber with brain slices was placed in a water bath and temperature was gradually increased to $35^{\circ} \mathrm{C}$. Afterward, the temperature of the water bath was maintained at $35^{\circ} \mathrm{C}$ for $45 \mathrm{~min}$ ("recovery"). After recovery, slices were maintained at room temperature in sucrose aCSF for the rest of the day (Figure 1A). All aCSF for slicing and recording was constantly oxygenated using carbogen $\left(95 \% \mathrm{O}_{2}\right.$ and $\left.5 \% \mathrm{CO}_{2}\right)$.

Brain slices were transferred from the holding chamber to a recording chamber for data collection. Two types of recording chambers were used in the current study: a "classic" submerged chamber (classic chamber, RC-26GLP, Harvard Apparatus, Figure 1B) and a chamber we call "interface-like" (Figure 1B). The interface-like chamber is a submerged chamber but flow is both above and below the slice, making it different from the classic chamber where flow is only above the slice. For this reason the chamber that we call interface-like is more like an interface chamber and it is often called that (interface-like chamber, RC27LD, Harvard Apparatus, Figure 1B). When slices were placed in the recording chamber, we used aCSF with $0 \mathrm{mM} \mathrm{MgSO}_{4}$ and $5 \mathrm{mM} \mathrm{KCl}\left(0 \mathrm{Mg}^{2+} / 5 \mathrm{~K}^{+}\right.$aCSF$)$as follows (in $\left.\mathrm{mM}\right): 130 \mathrm{NaCl}$, $5 \mathrm{KCl}, 1.25 \mathrm{NaH}_{2} \mathrm{PO}_{4}, 25 \mathrm{NaHCO}_{3}, 10 \mathrm{D}$-glucose, $0 \mathrm{MgSO}_{4}$, and $2.4 \mathrm{CaCl}_{2}$. Recordings were performed at $32 \pm 1{ }^{\circ} \mathrm{C}$ with 5-7 $\mathrm{mL} / \mathrm{min}$ flow rate.

It should be noted that recording at $32^{\circ} \mathrm{C}$ was chosen because higher temperature led to reduced viability, particularly in the

TABLE 1 | Conditions tested for the development of SD and SLEs using $0 \mathrm{Mg}^{2+} / 5 \mathrm{~K}^{+}$aCSF.

\begin{tabular}{|c|c|c|c|c|c|c|c|}
\hline Species & Sex & \# of animals & Age (day) & Age range (day) & \# of slices & $\begin{array}{l}\text { Incidence of SD (\# slices with } \\
\text { SD/total \# slices tested) }\end{array}$ & $\begin{array}{c}\text { Incidence of SLE (\# slices } \\
\text { with SLE/total \# slices tested) }\end{array}$ \\
\hline \multicolumn{8}{|c|}{ Classic submerged style chamber } \\
\hline \multirow[t]{3}{*}{ Mouse } & $M$ & 2 & $26 \pm 2$ & $24-27$ & 8 & $1 / 8^{a}$ & $2 / 8$ \\
\hline & $\mathrm{F}$ & 2 & $27 \pm 1$ & $26-28$ & 8 & $2 / 8^{b}$ & $2 / 8$ \\
\hline & Total & 4 & $26 \pm 1$ & $24-27$ & 16 & $3 / 16^{c}$ & $4 / 16$ \\
\hline \multicolumn{8}{|c|}{ Interface-like submerged style chamber } \\
\hline \multirow[t]{3}{*}{ Mouse } & $M$ & 12 & $26 \pm 1^{e}$ & $20-30$ & 22 & $18 / 22^{a, d}$ & $14 / 22$ \\
\hline & $\mathrm{F}$ & 10 & $27 \pm 1$ & $21-36$ & 23 & $18 / 23^{b}$ & $8 / 23$ \\
\hline & Total & 22 & $26 \pm 1$ & $20-36$ & 45 & $36 / 45^{c}$ & $22 / 45$ \\
\hline Rat & $M$ & 10 & $35 \pm 3^{e}$ & $21-45$ & 20 & $0 / 20^{d}$ & $15 / 20$ \\
\hline
\end{tabular}

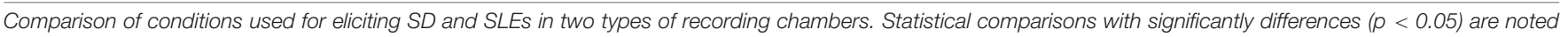
by superscript letters a-e. Tests were Fisher's exact (superscript a-d) or unpaired t-test (superscript e). 


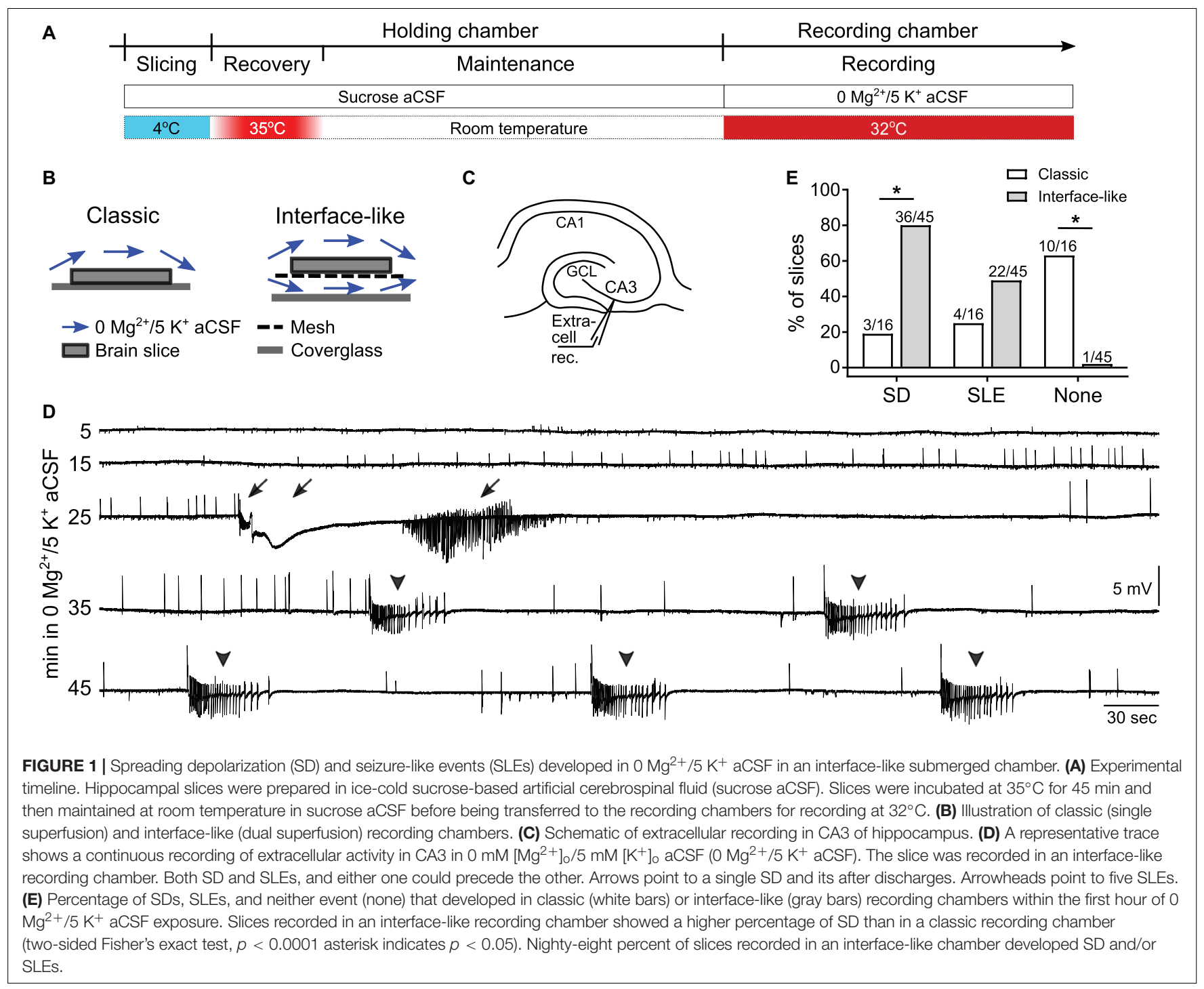

hilus of the DG. Ensuring viability in all parts of the slice was important to ensure that spread of SD was unaffected by tissue damage that can occur in slice preparation. In the past, when an interface recording chamber was used without infrared differential interference contrast (IR-DIC), recordings from different cell areas and cell types allowed us to judge viability. In the classic submerged or interface-like chamber, viability was addressed by confirming that the general morphology of cells prior to patch clamping was normal using IR-DIC at $40 \times$.

Notably, at $32^{\circ} \mathrm{C}, \mathrm{SD}$ and epileptiform activity may be less likely, and some aspects of SD such as cell swelling may be reduced because lower temperatures are protective. In rat slices, reducing temperature clearly inhibited SD caused by oxygen and glucose deprivation (Obeidat et al., 2000). On the other hand, when we tested temperatures from $30-35^{\circ} \mathrm{C}$ using an interface chamber without IR-DIC, electrophysiology was quite similar.

Recording electrodes were pulled (P-97, Sutter Instruments) from borosilicate glass $(1.5 \mathrm{~mm}$ outer diameter, $0.86 \mathrm{~mm}$ inner diameter, Sutter Instruments). An extracellular recording electrode containing $0 \mathrm{Mg}^{2+} / 5 \mathrm{~K}^{+}$aCSF (resistance 3-7 M $\Omega$ ) was placed in CA3 stratum pyramidale to record field potentials. For two simultaneous extracellular recordings, a second recording electrode was placed in either CA1 or the granule cell (GC) layer (GCL) at the crest of the dentate gyrus. In some slices, a second recording electrode (resistance 5-8 $\mathrm{M} \Omega$ ) was used to make whole-cell recordings of individual CA3 pyramidal cells simultaneously. The internal solution for whole-cell recording contained (in mM) $120 \mathrm{~K}$-gluconate, $10 \mathrm{HEPES}, 20 \mathrm{KCl}, 2 \mathrm{MgCl}_{2}$, 0.2 EGTA, $4 \mathrm{Mg}$-ATP, $0.3 \mathrm{Na}_{2}$-GTP, 7 Tris-phosphocreatine, and $0.5 \%$ biocytin. Data were amplified (MultiClamp 700B, Molecular Devices), digitized at $10 \mathrm{kHz}$ (Digidata1440A or 1550B, Molecular Devices), and acquired using pClamp (v. 10.7 or 11, Molecular Devices).

\section{Biocytin Labeling}

Immediately after recording, hippocampal slices were rinsed in $0.9 \%$ saline (all solutions used deionized water; $\mathrm{dH}_{2} \mathrm{O}$ ) and then preserved in $4 \%$ paraformaldehyde in $0.1 \mathrm{M}$ phosphate buffer 
( $\mathrm{pH}$ 7.4). Slices were stored at $4^{\circ} \mathrm{C}$ until they were processed. Procedures were conducted using free-floating sections at room temperature with mild agitation using a clinical rotator (The Belly Dancer, Stovall Life Science Inc.) unless otherwise specified. For the following steps, all buffers were prepared in $0.1 \mathrm{M}$ Tris buffer (TB, pH 7.6). First, slices were incubated in $1 \%$ TritonX 100 (Triton) for an hour to make cell membranes more permeable to reagents. Then endogenous peroxidase activity was suppressed by incubating slices in $0.1 \% \mathrm{H}_{2} \mathrm{O}_{2}$ for $30 \mathrm{~min}$. After three rinses (10 min each) with $0.25 \%$ Triton, slices were incubated in an avidin-biotin complex solution (ABC Elite Kit, Vector Laboratories) overnight at $4^{\circ} \mathrm{C}$ with gentle rotation. Slices were pretreated with $0.05 \% 3,3^{\prime}$-diaminobenzidine (DAB) with $1 \mathrm{mM} \mathrm{NiCl}_{2}$ for $30 \mathrm{~min}$ followed by incubation of $0.05 \%$ DAB with $0.0075 \% \mathrm{H}_{2} \mathrm{O}_{2}$ (diluted in $\mathrm{dH}_{2} \mathrm{O}$ ) until biocytin-filled cells were clearly visualized. The DAB reaction was terminated by three $10 \mathrm{~min}$ washes in TB. Slices were equilibrated with increasing concentrations of glycerol (15 min each, 25\%, 40\%, $55 \%, 70 \%, 85 \%, 100 \%$ ) (Hamam and Kennedy, 2003). Slices were coverslipped in $100 \%$ glycerol and edges of coverslip were sealed using nail polish. Brightfield images were taken using a charge-coupled device (CCD) camera (Retiga R2000, Teledyne QImaging) and an upright microscope (BX61, Olympus) with a $10 \times$ objective (UPlanSApo, 0.40 N.A., Olympus). ImagePro 7 Plus (Media Cybernetics) software was used for image acquisition. Brightfield images from different focal plans were stacked using CombineZP software (Hadley, 2010; Botterill et al., 2017) or the Stack Focuser plug-in in ImageJ (Umorin, 2002; Schneider et al., 2012).

\section{Intrinsic Optical Imaging}

Spontaneous SD and SLEs in CA3 were continuously monitored using field potential recordings. Recording of intrinsic optical signals was started manually as soon as electrophysiological manifestations of SD were observed. The time of the beginning of the imaging was recorded and matched with electrophysiological recordings. One slice was excluded from imaging analysis because the imaging was started after the beginning of SD.

Intrinsic optical signals were captured using continuous white light (halogen bulb) and a $775 \mathrm{~nm}$ band pass filter. Time-lapse images were taken at one or two frames per second. All images covered each of the major subfields in hippocampus (dentate gyrus, CA3, CA2, CA1, subiculum) and some of the cortex. The first image of an event was used as a baseline for comparisons of changes in intrinsic optical signals within the event. This method to define a baseline was chosen because the first three images showed minimal or no change in intrinsic optical signals.

Time-lapse images were acquired every $0.5 \mathrm{~s}$ for 11 slices and $1 \mathrm{~s}$ for 2 slices. The overall propagation patterns of imaged SDs were similar whether acquisition was 0.5 or 1 s/image, so the data using the two different acquisition rates were pooled. A CCD camera (Retiga Electro, Teledyne Photometrics) mounted on an upright microscope (BX51, Olympus) with a $4 \times$ objective was used for image acquisition. No multiplier was used between objective and the camera. A $775 \mathrm{~nm}$ filter was used to allow red/far red light to pass through the recorded slice. The light intensity was determined at the time when a slice was transferred into the recording chamber without saturated pixels. The same light intensity was used throughout the recording of a given event. Exposure time of each image was $10 \mathrm{~ms}$. MicroManager (Edelstein et al., 2010, 2014) or Ocular (Teledyne Photometrics) was used for image acquisition. Images were stored in stacks and analyzed using ImageJ or Fiji (Schindelin et al., 2012; Schneider et al., 2012).

To visualize changes in light transmission in pseudocolor, several steps were taken. First, images during an event (SD or SLE) were subtracted from the baseline image. Specifically, Image Calculator in ImageJ was used to calculate the difference between an image stack of an event (Event Stack) and an image stack generated from the event's baseline image (Baseline Stack). The result was a stack of images that showed the difference from the baseline in light intensity (Result Stack). Next, images from Result Stack were pseudocolored for better visualization where warmer and cooler colors indicated higher and lower light transmission, respectively. The Event Stack was later combined with its Result Stack side by side for presentation (Combined Stack). The Combined Stack was saved as a video file and replayed to review the propagation of SD across hippocampal subfields (Supplementary Video 1).

Changes in light transmission were quantified using ImageJ. The quantification procedure is illustrated in Supplementary Figure 3. In short, a square box of 50 by 50 pixels was used to sample part of a cell layer of a subfield. The box size was chosen so that it enclosed a representative part of the cell layer with minimal overlap with adjacent layers. Each box was a region of interest (ROI) that was marked and added to the ROI Manager function in ImageJ. These regions included the CA3 cell layer (at or close to the electrophysiological recording site), GCL upper blade, GCL crest, GCL lower blade, and CA1. The Multi Measure function was used to obtain the mean gray value of each ROI (Supplementary Figure 3). Data were visualized in RStudio (v. 1.1.456 ${ }^{1}$ ).

\section{Calculation of Spreading Depolarization Propagation Speed}

Spreading depolarization propagation speed was calculated by dividing the length of the SD propagation path (in $\mathrm{mm}$ ) by the timespan (in min) between two ROIs. An SD propagation path was drawn based on the observations of optical imaging. Because $\mathrm{SD}$ propagated from CA3 to CA1 like a wave, and the path followed the curvature of the pyramidal cell layer, the distance between CA3 and CA1 was measured along the curved cell layer and used to calculate the propagation speed. The distance between CA3 and the crest of GCL was measured in a different way because the wave front shown in the intrinsic optical images appeared to first move from CA3 to the upper blade in a linear path and then from upper to lower blade in a path following the curvature of the GCL. Therefore, the distance between CA3 and the crest of GCL was defined as the sum of two segments: (1) a straight line between the origin in CA3 and the tip of upper blade, and (2) a curved line along the GCL from the tip of upper blade to the crest. A timespan between two ROIs were determined

\footnotetext{
${ }^{1}$ http://www.rstudio.com/
} 
by the number of images and the image sampling frequency (every 0.5 or $1 \mathrm{~s}$ ).

\section{Quantification}

Electrophysiological recordings were quantified using Clampfit software (v. 10.7 or 11.1.0.23, Molecular Devices). Supplementary Figure 1A illustrates how an SD was measured. When an SD was observed, its onset was the beginning of the train of bursts just before the DC negative shift. As mentioned above, a burst consisted of at least one population spike superimposed on a positivity. A $5-10 \mathrm{~s}$ period before the beginning of the train of bursts was defined as the baseline. The amplitude of an SD was measured from the baseline to the maximal negative deflection. Additional synchronized activity that occurred during the end of the recovery phase, also called afterdischarges, contained negative-going, fast spike-like events in extracellular recordings. This additional synchronized activity was documented in a binary fashion, i.e., slices either had or did not have them. SD half duration was the time from the onset of the negative DC shift to the timepoint when the recovery reached $1 / 2$ of the peak amplitude of the negative DC shift.

Supplementary Figure 1B shows how SLEs were quantified. Similar to an SD, the onset of a SLE was the beginning of the train of bursts. There was no large DC negative shift like SD but there could be a small slow DC shift superimposed by bursts during a SLE. The Amplitude of this small DC shift during a SLE was defined by the difference between the baseline potential and the most negative deflection. Population spikes were not considered in this measurement. A 5-10 s period before the beginning of the train of bursts was defined as the baseline. The duration of a SLE was the onset of the first burst to the time when the last burst ceased.

\section{Statistical Analysis}

Statistical analyses were performed using Prism (GraphPad) or RStudio (v. 1.1.463, see text footnote 1). Data were first examined for their normality using the Shapiro-Wilk test. Homogeneity of variance was tested using the $F$ test. An unpaired $t$-test was used to compare means between two groups when data were normally distributed and without significant inhomogeneity of variance. When data were normally distributed with unequal variances, an unpaired $t$-test with Welch's correction was used. When data violated the assumption of normality, a Mann-Whitney $U$ test was conducted for comparison of two groups. Maximal light change of SD and SLE in CA3 and CA1 was analyzed using a two-way ANOVA with event type and subregion as betweensubject variables. Tukey's post hoc analysis was followed when the main test reached significance. The incidence of SD and SLE were compared using a Fisher's exact test. Differences were considered significant when $p<0.05$. Prism, RStudio, Inkscape (v. $0.92^{2}$ ), and GNU Image Manipulation Program (GIMP, v. 2.10.18 ${ }^{3}$ ) were used for preparation of figures.

${ }^{2}$ http://www.inkscape.org/

${ }^{3} \mathrm{http}: / /$ www.gimp.org

\section{RESULTS}

\section{An Interface-Like Chamber Promotes the Development of Spreading Depolarization and Seizure-Like Event in $0 \mathrm{Mg}^{2+} / 5 \mathrm{~K}^{+}$Artificial Cerebrospinal Fluid}

Spreading depolarization in area CA3 occurs in an interface chamber when aCSF contains $0 \mathrm{Mg}^{2+} / 5 \mathrm{~K}^{+}$(Mody et al., 1987; Scharfman, 1997). To examine SD in submerged mouse hippocampal slices, we first used a classic submerged chamber commonly used in patch clamp recording (Figure 1B). Recordings were made in area CA3 and used extracellular recording methods (Figure 1C) because this was done in prior studies using interface chambers. Many slices $(10 / 16$ or $63 \%$ of all slices tested, $\mathrm{n}=4$ mice) did not show any SD or SLE within $60 \mathrm{~min}$ of $0 \mathrm{Mg}^{2+} / 5 \mathrm{~K}^{+}$aCSF exposure. However, some slices exhibited SD (3/16) and some showed SLEs (4/16; Table 1).

Because most slices did not exhibit SD or SLEs, and previous reports showed that network activity is facilitated when aCSF flows above and below submerged slices (Hájos and Mody, 2009; Hájos et al., 2009; Morris et al., 2016), we tested an interfacelike chamber (Figure 1B) using the same slice preparation and recording methods. Figure $1 \mathrm{D}$ demonstrates a representative recording in the interface-like chamber. The recording shows the initial period of exposure (specifically from minute 5 to 50 after the start of exposure) of a mouse slice to 0 $\mathrm{Mg}^{2+} / 5 \mathrm{~K}^{+}$aCSF. In this example, one SD (arrow) and five SLEs (arrowhead) occurred. Typically, we found one SD and many SLEs (discussed further below). Almost all slices developed SD and/or SLEs (44/45 slices, 22 mice). There were significantly more slices that developed SD in the interface-like chamber than in the classic chamber (Fisher's exact test, $p=0.0009$; Figure 1E; Table 1).

In prior studies of SD in a submerged chamber, there is often an external stimulus to induce SD such as local application of high potassium or electrical stimulation (Lindquist and Shuttleworth, 2012; Steffensen et al., 2015). To the best of our knowledge, our results are the first to show SD in submerged slices using 0 $\mathrm{Mg}^{2+} / 5 \mathrm{~K}^{+}$aCSF.

\section{Characteristics of Spreading Depolarization and Seizure-Like Events in $0 \mathrm{Mg}^{2+} / 5 \mathrm{~K}^{+}$Artificial Cerebrospinal Fluid Spreading Depolarization}

In rat slices, $\mathrm{SD}$ did not develop in the interface-like chamber (20 slices, 10 rats, Table 1). Among mouse slices tested in the interface-like chamber, $80 \%$ of slices developed SD (36/45 slices, 22 mice; Table 1). The majority of slices showed one SD and the most SDs that were observed were three, and that was only found in one slice. The first SD developed after $33.6 \pm 1.4 \mathrm{~min}$ exposure of $0 \mathrm{Mg}^{2+} / 5 \mathrm{~K}^{+}$aCSF (36 slices, 19 mice). In the slice that developed three SDs, the intervals between onsets of SDs were 12.1 and $13.3 \mathrm{~min}$. The time between the onset of SD and the 
beginning of the characteristic negative DC shift was $10.7 \pm 0.8 \mathrm{~s}$ (36 slices, 19 mice).

The negative DC shift consisted of one or two peaks. Seventyseven percent of slices that developed SD showed two peaks (27/35 slices, 18 mice; 1 slice was not included due to a technical problem that occurred during the recording). The time from the beginning of the negative DC shift to the first peak was $2.2 \pm 0.2 \mathrm{~s}$ and to the second peak was $22.6 \pm 1.9 \mathrm{~s}$ (27 slices, 18 mice). The peak amplitude of the first and the second peaks were $-3.1 \pm 0.4$ and $-3.5 \pm 0.4 \mathrm{mV}$, respectively (27 slices, 18 mice). The amplitude difference between the two peaks was mostly within one standard deviation with only three exceptions, where two slices had a larger second peak and one slice had a larger first peak. It is notable that these amplitudes were small relative to those recorded in rat slices from an interface chamber with similar electrophysiological equipment. There, SD may be $10 \times$ larger or more (Scharfman, 1997; Scharfman et al., 2003; Skucas et al., 2013) but the smaller size in submerged chambers is common for all field potentials, and attributed to the more limited current flow.

For slices that developed only one SD, the timespan from the beginning to the peak of negative DC shift was $22.6 \pm 12.7 \mathrm{~s}(8 / 35$ slices, 7 mice).

The half duration of SD was $53.0 \pm 4.7 \mathrm{~s}$ (35 slices, 19 mice). During the recovery phase, 29/36 slices showed additional synchronized activity. The synchronized activity lasted for $102.9 \pm 6.1 \mathrm{~s}$ ( 29 slices, 17 mice). No sex difference was observed in the measurements of incidence of SD, number of SD, SD onset, and maximal SD amplitude (Supplementary Figure 2).

In a subset of experiments, simultaneous whole-cell recording was performed to examine the activity of individual CA3 pyramidal cells (PCs) during SD (five cells, five mice; Figure 2A). Recorded cells were filled with biocytin, and the cell type was confirmed later to have the morphological characteristics of CA3 PCs, such as a prominent apical dendritic tree, basal dendrites, and spiny dendrites (Lorente De Nó, 1934; Figure 2B). The electrophysiological characteristics also were consistent with a PC, such as an AP with a time course similar to a "regular spiking" neuron and an absence of the characteristic large afterhyperpolarizations of GABAergic neurons (Scharfman, 1993a,b, 1995).

In the five recorded PCs, a sudden, large depolarization that occurred at the start of SD was $21.5 \pm 2.4 \mathrm{mV}$. After the cessation of cell firing, a second larger depolarization of $39.5 \pm 3.7 \mathrm{mV}$ followed. Therefore, the peak of the depolarizations was close to $0 \mathrm{mV}$. As expected for $\mathrm{SD}$, the depolarization phase corresponded to the negative DC shift observed in the extracellular recording (Figure 2C). Cells repolarized and then hyperpolarized for $7.4 \pm 3.2 \mathrm{mV}$ before returning to their resting membrane potentials. Additional synchronized activity observed in extracellular recordings corresponded to large amplitude depolarizations and/or AP firing (Figure 2C).

\section{Seizure-Like Events}

In 45 tested mouse slices, $49 \%$ of slices developed SLEs within the $60 \mathrm{~min}$ of exposure to $0 \mathrm{Mg}^{2+} / 5 \mathrm{~K}^{+}$aCSF (22/45 slices, 22 mice; Table 1). Among 22 slices that showed SLEs, 8 of them developed only SLEs and the other 14 slices developed both SD and SLEs. More SLEs developed in slices without an SD (without SD, 8 slices, $10 \pm 1$ SLEs; with SD, 14 slices, $5 \pm 1$ SLEs; unpaired $t$-test, $t(20)=2.743, p=0.01$ ), suggesting a negative interaction between SD and SLEs.

The average duration of SLEs was $41.4 \pm 4.0 \mathrm{~s}$ (22 slices, 14 mice). Although the duration of SLEs was indifferent with or without an SD (without SD, 8 slices, $42.8 \pm 2.8 \mathrm{~s}$; with SD, 14 slices, $40.7 \pm 6.2 \mathrm{~s}$; unpaired $t$-test with Welch's correction, $\mathrm{t}$ $(17.42)=0.3069, p=0.76)$, SLEs with SD showed greater variation than SLEs without SD ( $F$ test, $F=8.8, p=0.0079$ ). This result supports the idea mentioned above that SDs interfere with SLEs.

During SLEs, an extremely small negativity developed. The average negativity was $-0.7 \pm 0.1 \mathrm{mV}$ (21 slices, 14 mice). The average negativity of SLEs did not appear to depend on appearance of SD (without SD, eight slices, $-0.7 \pm 0.1 \mathrm{mV}$; with $\mathrm{SD}, 13$ slices, $-0.7 \pm 0.1 \mathrm{mV}$; unpaired $t$-test, $t(19)=0.2455$, $p=0.81$ ).

For seven cells in five mice, intracellular recordings were made simultaneous to extracellular recordings and showed the components of SLEs (Figure 2D). The trains of bursts during SLEs were qualitatively similar to the initial train of bursts at the onset of SD (Figure 2C). However, the duration of the train of bursts of an SLE was longer than the train of bursts at SD onset (SLEs, $40.9 \pm 4.2 \mathrm{~s}$, 21 slices, 13 mice; train of bursts at SD onset, $9.3 \pm 0.8 \mathrm{~s}, 33$ slices, 18 mice; Mann-Whitney test, $U=3$, $p<0.001)$. The similarity in the trains of bursts of SLEs and SDs suggest that an SLE was a failure of bursts to trigger SD.

\section{Comparisons Between Spreading Depolarization and Seizure-Like Events \\ Incidence}

During the first $60 \mathrm{~min}$ of exposure to $0 \mathrm{Mg}^{2+} / 5 \mathrm{~K}^{+}$aCSF, 31\% of mouse slices developed both SD and SLE (14/45 slices, 22 mice) while $49 \%$ of slices developed only SD (22/45 slices), and $18 \%$ of slices developed only SLEs (8/45 slices). The differences were significant, with more slices exhibiting SD than SLE (SD, 36/45 slices; SLE, 22/45 slices, Fisher's exact test, $p=0.0039$, Figure 2E1). Each slice developed significantly fewer SDs than SLEs (SD, $0.8 \pm 0.1$; SLE, $3.2 \pm 0.7 ; 45$ slices, Wilcoxon matched pairs signed rank test, $p=0.049$; Figure 2E2).

\section{Onset of Spreading Depolarization and Seizure-Like Event}

The first SD occurred $33.6 \pm 1.4 \mathrm{~min}$ after the slice was transferred into the recording chamber with $0 \mathrm{Mg}^{2+} / 5 \mathrm{~K}^{+}$aCSF (36 slices, 22 mice), which was not different from the first SLE $(37.1 \pm 2.4 \mathrm{~min}, 22$ slices, 21 mice; unpaired $t$-test, $t(56)=1.348$, $p=0.18$; Figure 2E3). Among 14 slices that showed both SD and SLEs, SD was more likely to develop before a SLE (SD as the first event, 11/14 slices, SLE as the first event, 3/14 slices; Fisher's exact test, $p=0.0027)$. Thus, the onset of SD and SLE was similar, but an SD was more likely to be the first event in our $0 \mathrm{Mg}^{2+} / 5 \mathrm{~K}^{+}$ aCSF preparation.

\section{Amplitude and Half-Duration}

When the DC shift was measured (for Methods, see Supplementary Figure 1), SDs showed an average of a 4.7 times larger negative shift than SLEs $(\mathrm{SD},-3.3 \pm 0.4 \mathrm{mV}, 36$ 
A

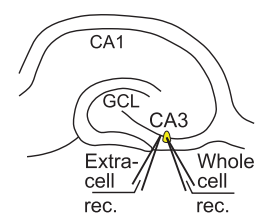

C SD Whole-cell recording
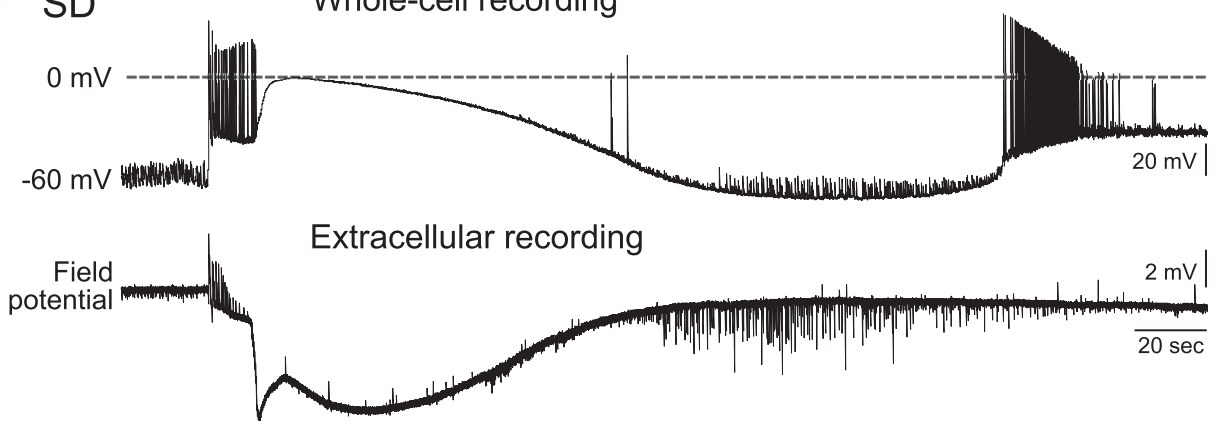

D SLE

B

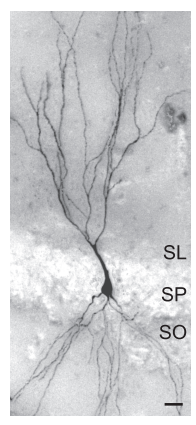

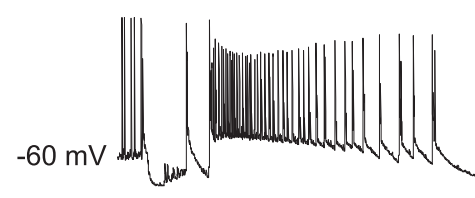

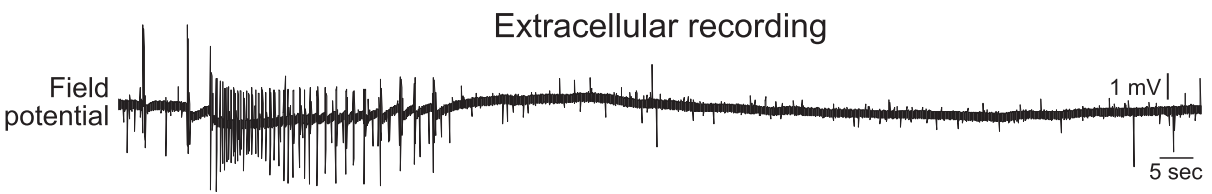

E

1 Incidence

2 Number of events

3 Onset
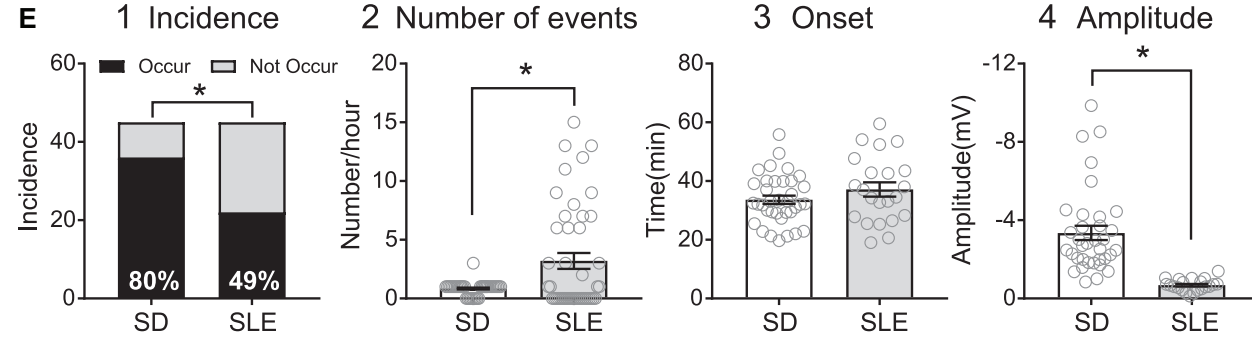

5 Duration

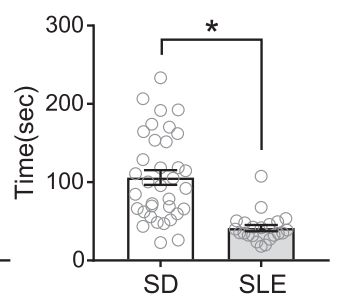

FIGURE 2 | SD and SLEs are two main types of events with distinct characters in the $0 \mathrm{Mg}^{2+} / 5 \mathrm{~K}^{+} \mathrm{aCSF}$ slice model. (A) Schematic illustrates the recording sites for simultaneous field potential and whole-cell recordings in CA3. (B) A representative image of a recorded and biocytin-filled CA3 pyramidal cell. SL, stratum lucidum; SP, stratum pyramidale; SO, stratum oriens. Scale bar, $20 \mu \mathrm{m}$. (C) Example of an SD. The intracellular and extracellular characteristics of SD are shown. (D) Example of a SLE. The intracellular recording shows characteristics such as a sudden depolarization and extensive firing. (E) Comparisons between SD and SLEs. (E1) In a 60-min recording period. SD showed a higher incidence than SLEs (two-sided Fisher's exact test, $p=0.02$; asterisk indicates $p<0.05$. (E2) In a 60-min recording period, more SLEs occurred than SDs (Wilcoxon test, $p=0.01$ ). (E3) The onset of the first SD and SLE was not significantly different (unpaired $t$-test, $p=0.18$ ). (E4) SD triggered a larger negative deflection than SLES (Mann-Whitney test, $p<0.0001$ ). (E5) SDs had a longer duration than SLES (Mann-Whitney test, $p<0.0001$ ). No sex difference was observed (Supplementary Figure 2).

slices, 19 mice; SLEs, $-0.7 \pm 0.1 \mathrm{mV}, 22$ slices, 14 mice; MannWhitney test, $U=12, p<0.0001$; Figure 2E4). When an SD's duration was estimated by doubling SD's half-duration, SD had duration more than twice the duration of SLEs (SD, $106 \pm 9$ s, 36 slices, 19 mice; SLE, $41 \pm 4$ s, 22 slices, 14 mice; Mann-Whitney test, $U=12, p<0.0001$, Figure 2E5). No sex difference was observed in these measures (Supplementary Figure 2).

\section{Spreading Depolarization Propagation Spreading Depolarization Spread to CA1 vs. Dentate Gyrus}

Propagation across gray matter is an important feature of SD. When $0 \mathrm{Mg}^{2+} / 5 \mathrm{~K}^{+}$aCSF was used in the past, it was suggested that $\mathrm{SD}$ that begins in CA3 appears to spread to
CA1 in hippocampus (Mody et al., 1987). Other studies using rabbit hippocampal slices and focal $\mathrm{K}^{+}$to elicit SD came to a similar conclusion (Haglund and Schwartzkroin, 1984). A spread to the dentate gyrus from CA3 through the upper blade has been reported when SDs are induced near CA2 using a local application of $\mathrm{K}^{+}$(Buchheim et al., 2002). Note the site is somewhat unclear because a diagram and text states CA1 but the depiction and images show CA2 or CA3a, depending on the septotemporal level, because the boundaries change with the septotemporal axis. However, spread from this general area to the dentate gyrus has not been studied before using $0 \mathrm{Mg}^{2+} / 5 \mathrm{~K}^{+}$ aCSF. Therefore, we first confirmed that the SDs we observed did spread and then asked how they spread. The site of origin of SD in our experiments was CA3 regardless of the septotemporal level. 


\section{Extracellular Recordings}

We began by electrophysiological recording in two locations using extracellular methods. First, we examined the propagation of SD from CA3 to CA1 in $0 \mathrm{Mg}^{2+} / 5 \mathrm{~K}^{+}$aCSF. Extracellular recording electrodes were placed in the $\mathrm{CA} 3 \mathrm{~b}$ (where onset of $\mathrm{SD}$ was exhibited) and CAlb regions (Figure 3A1). In seven slices, five developed an SD in CA3. In two of these slices, CA1 showed an SD. Consistent with past reports, the onset of the negative DC shift in CA1 was delayed from CA3 by an average of $8 \mathrm{~s}$ (range 5-11 s, Figure 3A2) whereas the initial train of bursts occurred at similar time $(<10 \mathrm{~ms})$. These data are interesting because they suggest neuronal activity can occur with short a delays but the
A 1

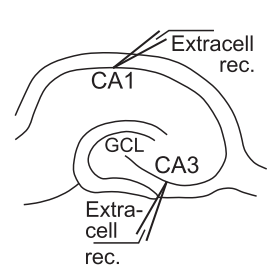

B

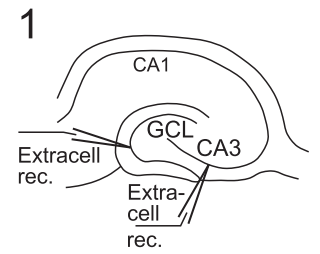

c 1

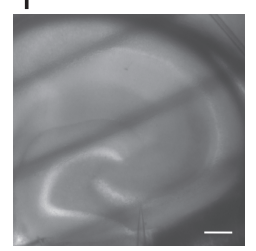
2

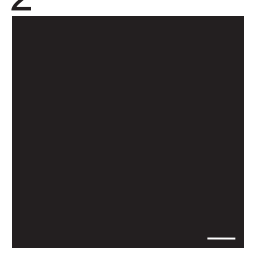

baseline

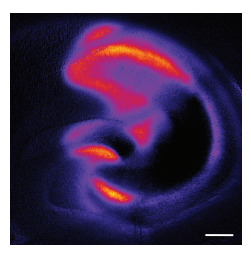

$20 \mathrm{sec}$

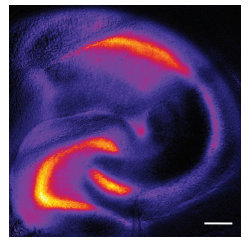

$70 \mathrm{sec}$
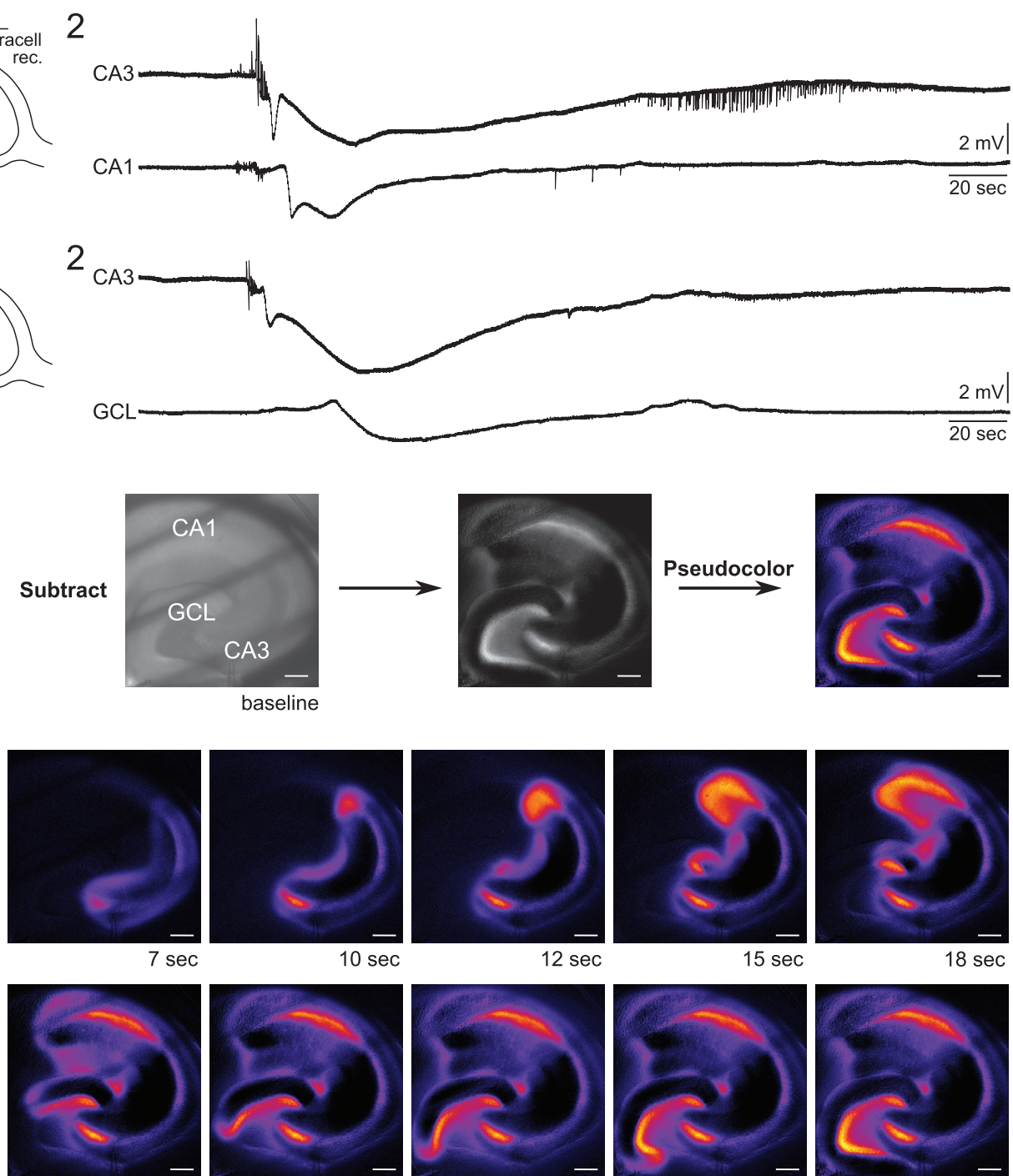

2
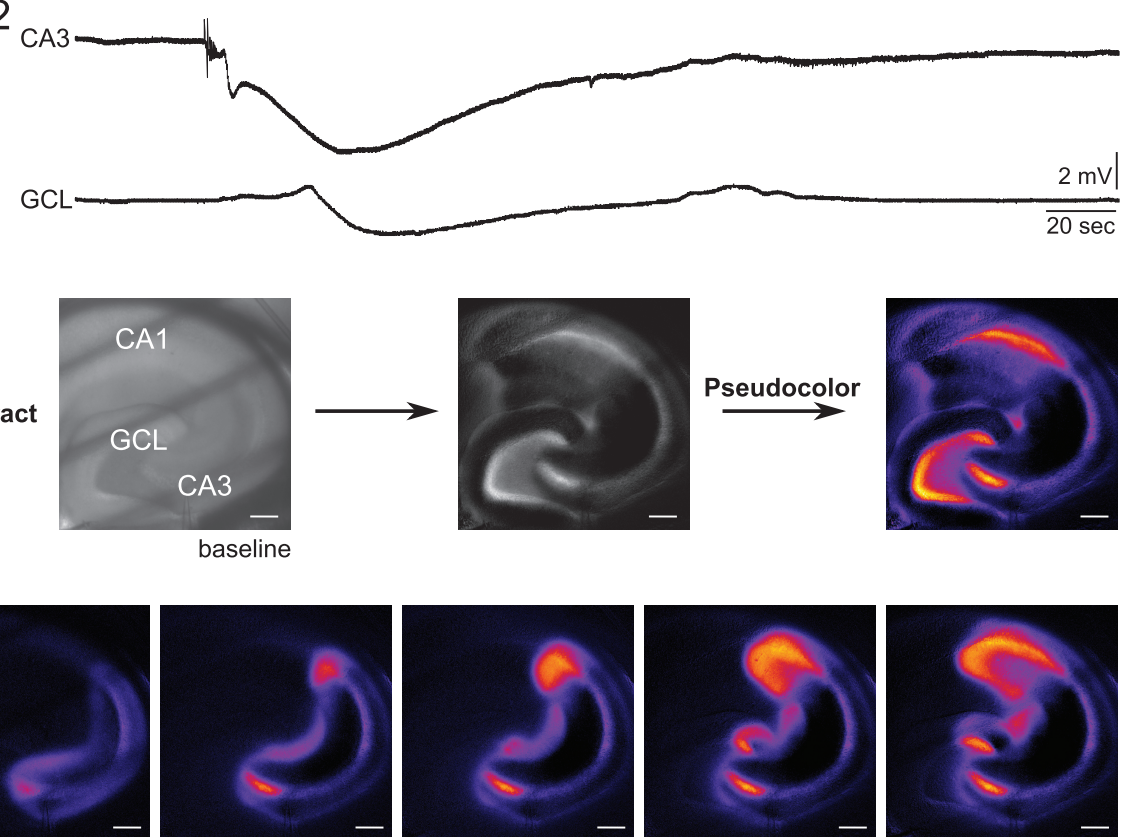

$10 \mathrm{sec}$
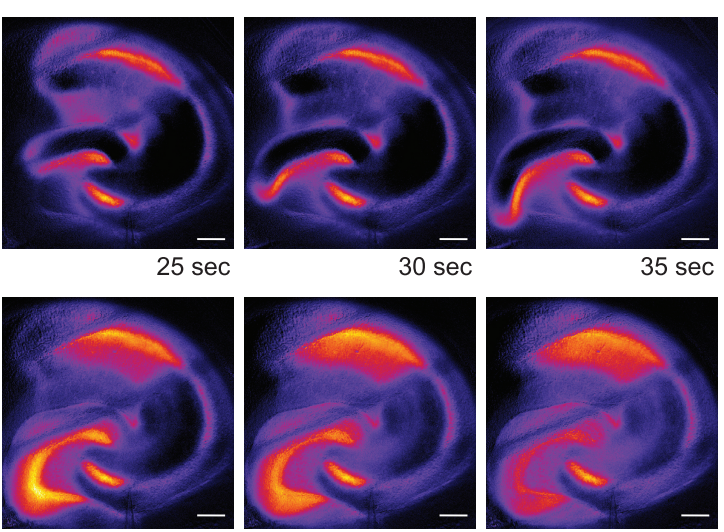

$90 \mathrm{sec}$

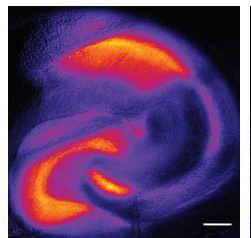

$120 \mathrm{sec}$
$35 \mathrm{sec}$

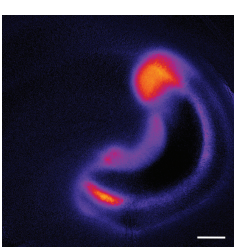

$12 \mathrm{sec}$

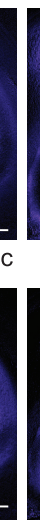

$150 \mathrm{sec}$

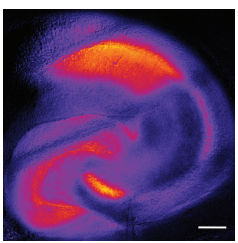

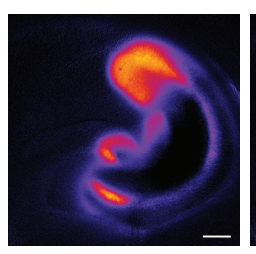

$15 \mathrm{sec}$

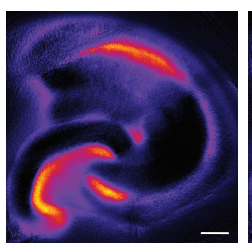

$40 \mathrm{sec}$

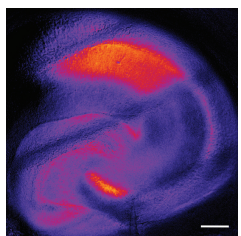

$180 \mathrm{sec}$
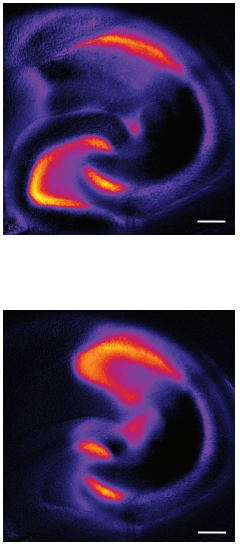

$18 \mathrm{sec}$

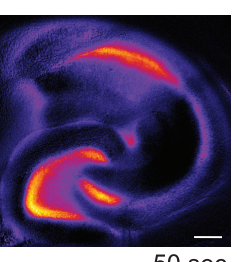

$\%$ Baseline

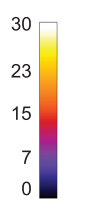

FIGURE 3 | Propagation of SD in mouse hippocampus. (A1) Schematic of dual field recordings in the CA3 and CA1. (A2) An SD propagated from CA3 to CA1. The electrical recordings show a significant delay between CA3 and CA1 DC shift, corresponding to the delay in the optical image in (C). (B1) Schematic of dual field recordings in the CA1 and the GCL. (B2) An SD propagated from CA3 to the GCL with a delay. (C1) An SD was accompanied by a changed in the intrinsic optical signal. The difference in light transmission was calculated by subtracting a baseline image from the image acquired during SD. (C2) Consecutive images illustrate the propagation of an SD. Diagonal dark lines were from the mesh underneath the brain slice. GCL, granule cell layer. Scale bar, $200 \mu \mathrm{m}$. 
large cell depolarization, occurring during the negative DC shift, is first in CA3 and very slowly propagates to CA1.

Next, we examined the propagation of SD from CA3 to the dentate gyrus. Extracellular recording electrodes were placed in the CA3 PCL and GCL at the crest of the dentate gyrus, which was chosen because it is the furthest point from CA3 in the GCL (Figures 3B1,B2). In two slices with these recording locations, both slices showed SD in the GCL with a long delay of $26.7 \mathrm{~s}$ (range 24.9-28.5 s, two slices from two different mice). Our data suggest that $\mathrm{SD}$ that develops in CA3 during superfusion of 0 $\mathrm{Mg}^{2+} / 5 \mathrm{~K}^{+}$aCSF can propagate to both CA1 and dentate gyrus and with different delays, and the delay from CA3 to the dentate gyrus appears to be longer than the delay from CA3 to CA1.

\section{Intrinsic Optical Imaging}

Spreading depolarization-induced neuronal swelling changes the light transmission. Specifically, the light transmission increases when an upright brightfield microscope is used (Figure 3C1, Buchheim et al., 2002; Mané and Müller, 2012). We used this characteristic of SD to examine the path of the SD wavefront across hippocampal subregions in our slice preparation.

To capture changes in light transmission that reflected SD propagation, images of SDs were taken and subtracted from the baseline image (see Methods and Figure 3C1). Figure 3C2 shows images selected in temporal order throughout an SD. In 9/11 imaged slices the wave fronts of the SDs all emerged from CA3 (Figure 4). SDs of the exceptional two slices were not fully imaged so the emergence of their wavefronts was unclear. Within the nine slices, $44 \%$ of SDs propagated into both CA1 and the whole dentate gyrus (4/9 slices). Other SDs propagated either only into the whole dentate gyrus (2/9 slices), or only into hilus (2/9 slices), or only into CA1 (1/9 slices). The direction of SD propagation was either toward CA1 or toward the dentate gyrus or both. No association between propagation pattern and dorsoventral axis of hippocampus was observed.

To quantify the propagation speed of SDs, we measured the length of the SD traveling path among our defined regions of interests (see section "Calculation of Spreading Depolarization Propagation Speed") and the time that elapsed from the start of the path to its end. The propagation speed from CA3 to CA1 was $4.4 \pm 1.6 \mathrm{~mm} / \mathrm{min}$ (range $2.6-8.6 \mathrm{~mm} / \mathrm{min}$, five slices, three mice). The propagation speed from CA3 to the crest of the GCL in the dentate gyrus was $2.7 \pm 0.5 \mathrm{~mm} / \mathrm{min}$ (range 1.8-4.6 $\mathrm{mm} / \mathrm{min}$, nine slices, four mice). Propagation speed from CA3 to CA1 was not statistically different from the speed from CA3 to GCL [unpaired $t$-test with Welch's correction, $t(4.706)=2.284, p=0.07]$.

The Supplementary Video $\mathbf{1}$ shows the emergence, propagation, and recovery of an SD using original brightfield and pseudocolored images. Taken together, these results show that most SD wave fronts emerge from CA3 in $0 \mathrm{Mg}^{2+} / 5 \mathrm{~K}^{+}$aCSF. $\mathrm{SD}$ then spreads both in the direction of the trisynaptic circuit, i.e., to CA1, and in the opposite direction of the trisynaptic circuit, i.e., to the dentate gyrus. The speeds are similar and consistent with past studies (Obeidat and Andrew, 1998; Buchheim et al., 2002).

\section{Spreading Depolarization Spread From Upper to Lower Blades of the Dentate Gyrus}

When SDs in CA3 propagated toward the dentate gyrus, $25 \%$ of SDs (3/12 slices, 6 mice) reached the hilus and/or upper blade of GCL but then stopped spreading to the other parts of the dentate gyrus. The rest of the SDs (9/12 slices, 6 mice) traveled to the upper blade and then spread to the crest and finally the lower blade (Figure 5A).

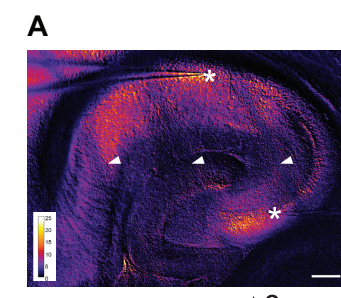

$\Delta 0 \mathrm{sec}$

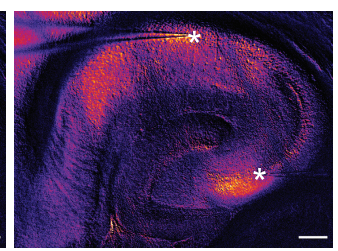

$\Delta 0.5 \mathrm{sec}$

B

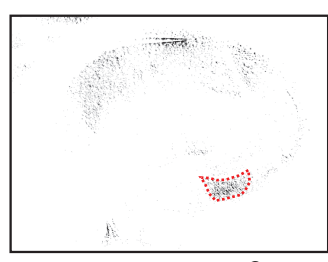

$\Delta 0 \mathrm{sec}$

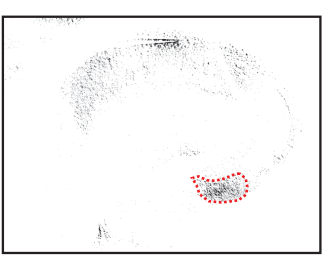

$\Delta 0.5 \mathrm{sec}$

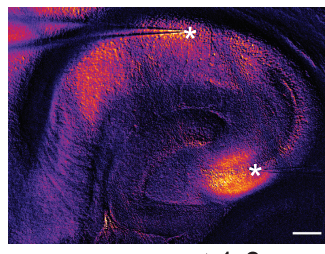

$\Delta 1.0 \mathrm{sec}$

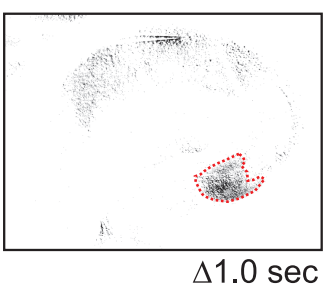

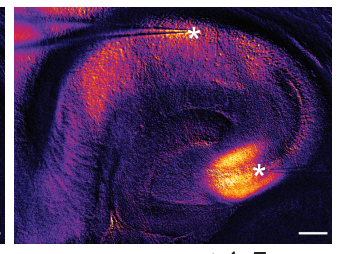

$\Delta 1.5 \mathrm{sec}$

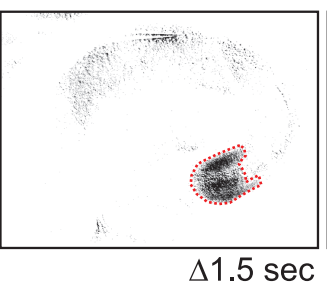

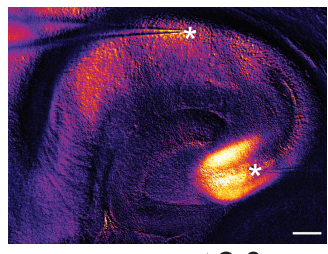

$\Delta 2.0 \mathrm{sec}$

FIGURE 4 | The initial wave of SD often begins in CA3 in $0 \mathrm{Mg}^{2+} / 5 \mathrm{~K}^{+}$aCSF. (A) Time lapse images show the initiation site of an SD in CA3. The images during SD were first subtracted from a baseline image and then pseudocolored. Warmer color indicates higher light transmission and cooler color reflects lower light transmission. The increase in light transmission began in both SP and SL of CA3 before a wave front became prominent. Stars: recording sites in CA3 and CA1. Arrowheads: dark areas reflecting the mesh underneath the slice. Scale bar, $200 \mu \mathrm{m}$. (B) Same images from panel (A) were thresholded so that areas with the greatest change in light transmission in CA3 could be distinguished (dashed red lines). 


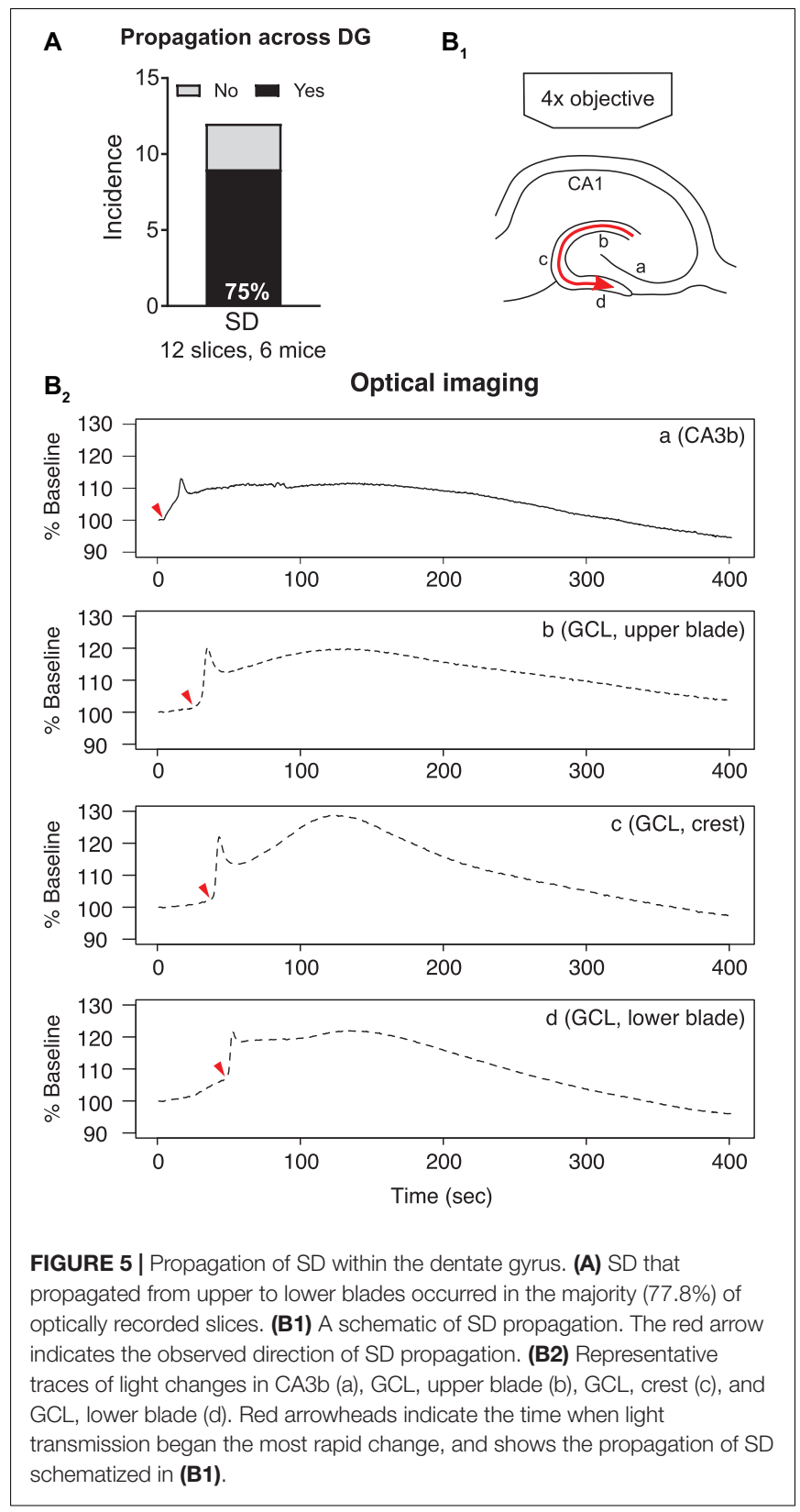

Spreading depolarizations we observed entered the dentate gyrus in two separate waves: into the hilus and into the lateral tip of upper blade (Figure 3C2). Although the lateral tip of lower blade of GCL was also approached by the wave front (Figure 4), it seemed to occur with entry into the hilus, which spread into the entire area between both blades rapidly. The wave front stopped at the boundary of hilus with the GCL. The wave front that entered the upper blade moved through the GCL and molecular layer together and followed the curvature of the GCL so that it traveled from upper blade to crest to lower blade in a slow continuous wave (Figure 5B1).

Quantification of light transmission supported the propagation pattern of SD wave front. The onset of sharp rise of light transmission happened in sequence among
CA3b (Figure 5B2a), GCL upper blade (Figure 5B2b), GCL crest (Figure 5B2c), and GCL lower blade (Figure 5B2d). Interestingly, after the first peak in light transmission there was a short drop of light transmission followed by an increase of light transmission in the GCL and molecular layers (Figure 5B2, Supplementary Video 1). This secondary increase in light transmission was also observed in CA3 and CA1, and the underlying cellular correlates remain unclear.

To quantify the speed of propagation within the dentate gyrus, we measured the length of SD propagation by following SD's traveling path, and the time spent from upper blade, crest to lower blade (see section "Calculation of Spreading Depolarization Propagation Speed"). The speed of propagation was $2.8 \pm 0.5 \mathrm{~mm} / \mathrm{min}$ (range $1.4-4.0 \mathrm{~mm} / \mathrm{min}$, seven slices, four mice).

\section{Seizure-Like Events Showed Increased but Weak Intrinsic Optical Signals}

Next, we quantified changes in light transmission of SLEs. Figure 6 compares the change in light transmission of SDs to SLEs. In contrast to SD (Figure 6A2), the change in light transmission during the SLEs was minimal (Figure 6B2. However, although changes in light transmission in SLEs were small, they were significantly greater than baseline [one sample $t$-test, CA3, $t(6)=4.75, p=0.0032$; CA1, $t(6)=3.96, p=0.0075$ ].

It was not surprising that the percent change of maximal light transmission of SD was greater than SLEs [two-way ANOVA, SD vs. SLEs, $F(1,34)=12.69, p=0.0011$; CA3 vs. CA1, $F(1,34)=0.34$, $p=0.3376$; interaction, $F(1,34)=0.3585, p=0.5533$; Figure 6C]. This was true for CA1 (SD, $118.4 \pm 4.7 \%, 12$ SDs in 12 slices, 6 mice vs. SLEs, $104.0 \pm 1.0 \%$; 7 SLEs in 7 slices, 3 mice; $p=0.03$, Tukey's multiple comparison test) but not for CA3 (SD, $113.0 \pm 2.1 \%, 12$ SDs in 12 slices, 6 mice vs. SLEs, $102.7 \pm 0.6 \%$; 7 SLEs in 7 slices, 3 mice; $p=0.18$, Tukey's multiple comparison test). These data suggest that electrical recordings are quantitatively more sensitive than light transmissions.

In summary, SLEs showed a very small but detectable change in light transmission. The data are consistent with studies showing an increase in light transmission associated with an increase in neuronal activity (MacVicar and Hochman, 1991; D’Arcangelo et al., 2001).

\section{DISCUSSION}

\section{Summary}

In the present study, we first addressed methods to use mouse slices to study SD and SLEs during exposure to $0 \mathrm{Mg}^{2+} / 5 \mathrm{~K}^{+}$ aCSF. We made several new insights (Table 2 ). We recorded these events using extracellular recording, whole-cell recording, and intrinsic optical imaging, which was advantageous both temporarily and spatially. We found that the chamber mattered: SD was more likely to develop in submerged mouse slices in an interface-like than in a classic submerged chamber. Species also mattered: mouse hippocampal slices developed SD and rat slices did not show SD. However, both species showed SLEs. Notably, 
A 1

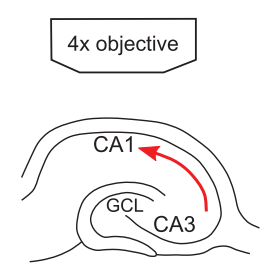

2

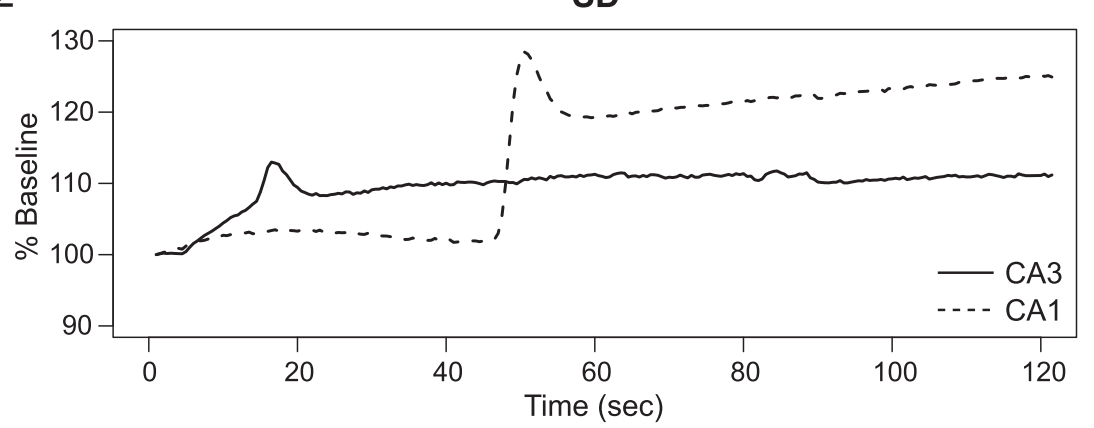

B 1

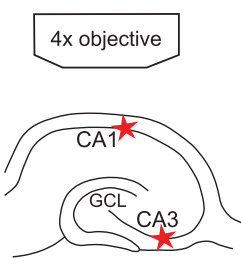

2

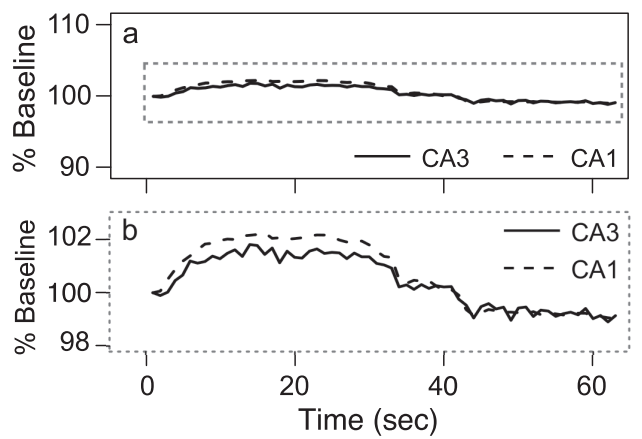

C
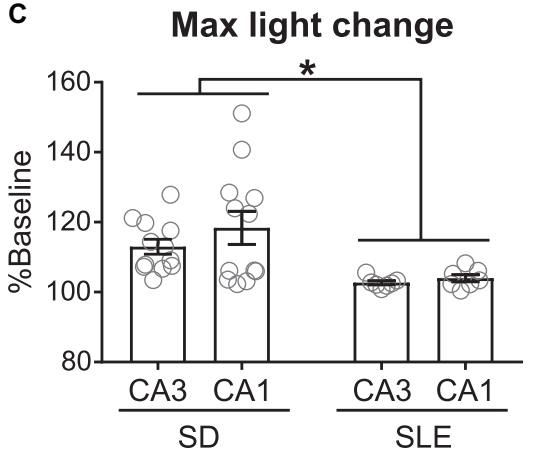

FIGURE 6 | SDs but not SLEs, show prominent changes in light transmission. (A1) Schematic of the path of light transmission in panel A2. Arrow indicates the propagation of the observed SD. (A2) Change of light transmission initiated in CA3 (solid line) and then followed by CA1 (dashed line) with a delay. (B1) Schematic of the region of interests (stars) in panel B2. (B2) A SLE showed subtle changes in light transmission (expanded in b) without a long delay between CA3 (solid line) and CA1 (dashed line). (C) SDs had significantly higher changes in light transmission than SLEs [two-way ANOVA, SD vs. SLEs, $F(1,34)=12.69, p=0.0011 ;$ CA3 vs. CA1, $F(1,34)=0.34, p=0.3376$; interaction, $F(1,34)=0.3585, p=0.5533]$.

TABLE 2 | Summary of new insights.

- Spontaneous SD following exposure to $0 \mathrm{Mg}^{2+} / 5 \mathrm{~K}^{+}$aCSF in submerged mouse slices occurs in the majority of slices but occurs only once or twice in the first hour of recording. SD does not appear to depend on an immature age or sex.

- Trains of bursts or what we call SLEs occurred in a robust manner, usually after SD, and occurred repetitively for the first hour of recording. SLEs were stereotypical in morphology, and associated with a change in optical transmission, but optical transmission changes were minimal relative to SD.

- SD began with a flurry of bursts followed by the large negative DC shift that is typical of SD. The flurry of bursts was similar to the trains of bursts that comprised SLEs, suggesting they may be related. Upon recovery there were often trains of synchronized activity (usually called afterdischarges).

- The fact that SD occurred and usually SLEs followed suggests that SDs could delay, interfere, or inhibit SLEs. The fact that SLES occurred after SD and SD rarely repeated suggests that SLEs may have inhibited SD.

- Like focal stimulation to an area near CA2 (Buchheim et al., 2002), spread to the dentate gyrus from CA3 followed a path that typically involved slow invasion of the upper blade GCL and molecular layer from lateral tip to crest and then lower blade. Spread could also involve the entire hilar region, which has not been reported before. The optical transmission changes obeyed the GCL/hilar border in all cases, suggesting a novel aspect of the GCL/hilar border.

- Spread to CA1 from CA3 was delayed for the negative DC shift but minimal delay occurred for the initial electrical activity. In the dentate gyrus both were significantly delayed.

- Quantification of the optical transmission changes showed fluctuations during SD that were not observed by eye. These fluctuations varied from subfield to subfield, suggesting different dynamics of cell swelling that quantification can show better than the typical pseudocolor analyses.

A summary of new insights gained from the Results are listed. These are discussed in the "Results and Discussion" sections.

recording and imaging both SD and SLEs provided a chance to examine them in ways that have rarely been done in the past.

Intrinsic optical imaging allowed us to examine the emergence of the SD wave front and SD propagation without investing in additional dyes, transgenic mice, or equipment. Within hippocampus, we found that the majority of SDs initiated in CA3. Because of our imaging field only covered the hippocampus with limited cortical areas, we cannot exclude that SDs initiated in the small part of the cortex attached to the hippocampus (Martens-Mantai et al., 2014). Interestingly, when we recorded extracellular electrical activity in CA3 and CA1 simultaneously, the SDs began with a train of bursts with less than $10 \mathrm{~ms}$ delay between CA3 and CA1. However, the negative DC shift in CA3 immediately followed the train whereas in CA1 it was clearly delayed. The concurrent trains of bursts may have propagated synaptically from CA3 to CA1 but the drastic 
cell depolarization that underlies the DC shift relies on other mechanisms. Alternatively, the entorhinal cortex (EC) innervates both CA3 and CA1 pyramidal cells (Witter et al., 2017) and may trigger trains of bursts.

In hippocampus, SD propagated along the direction of classic trisynaptic circuit into CA1 and in the opposite direction into dentate gyrus. Among SDs that propagated into dentate gyrus, they traveled in a way that is not consistent with the idea that SD spreads in all directions by a simple process. Instead, SDs propagated in two separate waves-one moved into hilus and the other moved along molecular layer and GCL. The wave front that propagated into hilus obeyed the boundary between GCL and hilus, and it dissipated in hilus. The wave front that moved into molecular layer and GCL propagated from upper blade to crest, and then lower blade. The wave front encompassed the entire molecular layer without involving adjacent layers in CA1 or the hilus.

Other researchers have also studied the DG, although with different methods. Obeidat and Andrew (1998) found that oxygen and glucose deprivation led to changes in light transmission in CA1 and the DG at a similar time. In contrast, focal application of high potassium to an area near the CA1 border with CA2 led to more of a change in the adjacent CA1 only. One limitation was the area near the lower blade was cropped so it was not visible for the reader.

The results of oxygen and glucose deprivation were striking. Interestingly, their images of the DG suggest a distinct pattern of spread in some ways and similarities to our data in other ways. The differences included invasion first of the upper blade molecular layer, which was not fully invaded. At that time the GCL did not appear to have light transmission changes but after that time the GCL showed striking changes and did so throughout the upper blade GCL. This pattern is quite different from what we found, where both the molecular layer and adjacent part of the GCL showed optical transmission changes at the same time. Furthermore, there was initiation at the lateral tip of the upper blade and then movement to the center of the upper blade, border with the crest region, the crest, and finally the lower blade. Another difference was a general lack of invasion of the hilus when oxygen and glucose deprivation was studied. However, there was a general pattern of spread from upper to lower blade in both models of SD. The differences could be partly explained by generation of SD in CA1 by oxygen and glucose deprivation and generation in CA3 for our work. Also, lack of hilar participation could simply be due to a larger hilar area in our horizontally prepared sections compared to transverse sections used for oxygen and glucose deprivation. Hilar involvement would also be more likely with CA3 playing a major role in SD initiation because of the CA3 backprojecting axons to the hilus (Scharfman, 2007).

Another group also provided a beautiful study of how the DG is invaded by SD (Buchheim et al., 2002). Buchheim et al. (2002) used local pressure application of $1 \mathrm{M} \mathrm{KCl}$ in an area they stated was CA1 but could be CA2 or CA3a from the information in the figures. They found spread of SD into CA3 and then the upper blade, with subsequent spread toward the lower blade. The example that was shown had the lower blade clipped partially and at low power, so the hilus and GCL border were not entirely clear and some of the lower blade was absent entirely. There was late activity that could be part of the lower blade of the lower hilus - it is hard to say. One notable difference from our findings is invasion of the hilus seems absent except for the possibility of the late activity in the lower hilus. Our findings showed invasion could occur in the hilus extensively. The reason may be the septotemporal level of the slice, which was somewhat dorsal in the example of Buchheim et al. (2002). The backprojection from CA3 to the hilus is not as prominent in the dorsal hippocampus (Scharfman, 2007). Another noteworthy distinction is that the perforant path innervation of the DG is more robust in the horizontal plane in temporal sections (Witter et al., 2017). Dorsal sections transect the connections more. Thus, EC SD can spread to the DG readily in some ventral slices but not dorsally. Indeed, Buchheim et al. (2002) include a very nice diagram that shows spread from CA3 to the EC but no spread from the EC to the DG.

Our report is distinct in the ways described above, and the first one to show the SD propagation pattern in the DG using 0 $\mathrm{Mg}^{2+} / 5 \mathrm{~K}^{+}$aCSF.

The consistent direction of SD propagation across different SD induction models suggests that a common structural factor, e.g., the components of the neuropil like the extracellular matrix and vasculature which is not necessarily homogeneous. In particular, the GCL/hilar border has a very different milieu, which makes it subject to injury (Soltesz et al., 1995).

In addition to SD, SLEs developed in $0 \mathrm{Mg}^{2+} / 5 \mathrm{~K}^{+}$aCSF. SD and SLE had distinct characteristics: (1) the negative DC shift of SDs were more than $5 \times$ that of SLEs'; (2) SDs were approximately $6 \times$ longer than SLEs; and (3) SDs had approximately $7 \times$ larger changes in light transmission than SLEs. However, the trains of bursts underlying SLEs were quite similar to those before SD. These results suggest that once a SLE is initiated in CA3, it could complete as a SLE or trigger an SD. Possible factors that could switch the progression of a SLE into an SD may be related to the degree the slice preparation preserves the recurrent collaterals of CA3 PCs, which would allow greater synchrony and in turn, greater local accumulation of ions. The mossy fiber pathway may also be a factor, because it can vary in the degree it is maintained in a slice also; if well maintained its normally high concentration of glutamate can be released onto CA3 PCs to a greater extent, facilitating SD. Although our data do not suggest major variations in these factors, slices can vary from one to the next and from one animal to another.

Taken together, our optimized model with $0 \mathrm{Mg}^{2+} / 5 \mathrm{~K}^{+}$ aCSF led to a slow development of both SD and SLEs, which provides an opportunity to study these events without the typical trigger where the stimulus is immediately followed by SD. Additionally, our preparation also provided great temporal and spatial resolution by combining electrophysiological recording and optical imaging. We therefore think that this experimental model has many uses to advance our understanding of mechanisms of SD and interactions between SD and SLEs. Also, one could use the approach for validating preclinical drug candidates by taking advantage of a controlled ex vivo environment and relevance to disease mechanisms of migraine, TBI, stroke, etc. 


\section{The Importance of the Recording Chamber}

Submerged slices seem to be a suboptimal condition for SD and even the development of epileptiform activity when compared to slices situated at an interface of oxygenated solution and air. Thus, slices maintained in an interface chamber from rats showed both SD and epileptiform bursts in $0 \mathrm{Mg}^{2+}$ aCSF (3-5 $\mathrm{mM} \mathrm{K}^{+}$; Mody et al., 1987; Gloveli et al., 1995; Kovács et al., 1999), but only bursts were reported in submerged rat hippocampal slices in $0 \mathrm{Mg}^{2+}$ aCSF (2-5 $\mathrm{mM} \mathrm{K}^{+}$; Lewis et al., 1989, 1990; Neuman et al., 1989; Churn et al., 1991; Kojima et al., 1991; Billard et al., 1997; Wahab et al., 2009). When rat slices in a classic submerged chamber were compared with rat slices in an interface chamber, using experimental conditions that were otherwise the same, fewer submerged slices showed epileptiform bursts than slices in the interface chamber (Schuchmann et al., 2002).

Regarding anoxia-induced SD (hypoxia with normal glucose concentrations), SD occurred in an interface chamber but not in a classic submerged chamber (Croning and Haddad, 1998). Our data are consistent with these findings. On the other hand, when oxygen and glucose deprivation are used to induce SD, it has been shown that submerged slices exhibit SD (Juzekaeva et al., 2020; Grube et al., 2021).

Both SD and SLEs both have components of synchronized activity. An in vitro environment that supports synchronized activity would aid the development of SD and SLEs, and oxygen supply could be a critical player. The oxygen in interface chambers is supplied from carbogen-saturated humidified air and aCSF, while carbogen-saturated aCSF is the sole oxygen resource in submerged chambers. Since only a limited amount of oxygen can dissolve in aCSF, the direct contact with humidified air in an interface chamber has been considered as a more efficient way to supply oxygen than slices that obtain all oxygen from aCSF in a submerged chamber (Aitken et al., 1995). In interface chambers, rat brain slices showed in vivo-like rhythmic oscillations generated by synchronized cell activity (von Krosigk et al., 1993; Sanchez-Vives and McCormick, 2000; Case and Broberger, 2013). In submerged chambers, however, similar observations of synchronized activity seem possible only when oxygen supply is increased by superfusing aCSF above and below slices and using a fast flow rate (Hájos and Mody, 2009; Hájos et al., 2009). We found that the likelihood of developing SD in mouse slices significantly increased in an interface-like chamber, where $80 \%$ of mouse slices developed SD in an interface-like recording chamber when compared to $19 \%$ of slices in a classic recording chamber. Our results agree with previous findings that synchronous activity appears to be better supported in interface chambers. Furthermore, our results support the hypothesis that oxygen supply is important in SD and SLEs.

\section{Species Differences in the Development of Spreading Depolarization in 0 $\mathrm{Mg}^{2+} / 5 \mathrm{~K}^{+}$Artificial Cerebrospinal Fluid}

When mouse and rat slices were compared in the same preparation (interface-like chamber, fast flow, and $0 \mathrm{Mg}^{2+} / 5 \mathrm{~K}^{+}$ $\mathrm{aCSF}$ ), we found that SLEs were observed in slices from both species while SDs were exclusively in mouse slices, at least for SDs and SLEs following $0 \mathrm{Mg}^{2+} / 5 \mathrm{~K}^{+}$aCSF exposure. The discrepancy may be explained by the preservation of circuit components. We prepared both mouse and rat hippocampal slices using the same thickness $(350 \mu \mathrm{m})$. When using the same thickness, others have been proposed that mouse slices are likely to preserve more connections and circuit properties than rat slices (Heinemann et al., 2006). This argument is based on the idea that, as similar brain circuitry is compressed in the mouse relative to the rat, the compression would force more elements into a smaller volume in mouse compared to rat. In support, it was stated that epileptiform activity is more readily elicited in mouse than rat (Heinemann et al., 2006). However, our experience is distinct from Heinemann et al. (2006) because we do not necessarily find epileptiform activity more readily elicited in mouse. For example, when mouse slices were exposed to a buffer than increased excitability, the latency to spontaneous epileptiform activity in the EC was longer than rat (Duffy et al., 2015).

It is also possible that the rat slices need more oxygen because they are larger in surface area, despite the same thickness as the mouse slices. Another possibility to explain the species difference could be related to age. The ranges of ages overlapped but the youngest mice were younger than the youngest rats. On the other hand, we tested very young rats and mice (postnatal day 21-22) and we obtained similar results-rat slices did not exhibit SD, but mouse slices did. Therefore, age did not appear to be a factor in species differences.

\section{Spreading Depolarization vs. Seizure-Like Events in $0 \mathrm{Mg}^{2+} / 5 \mathrm{~K}^{+}$ Artificial Cerebrospinal Fluid}

In slices that developed both types of events, $73 \%$ of slices developed SD first. Most slices exhibited only one SD (32 of 33 slices; the exceptional slice had three SDs) in the first hour of $0 \mathrm{Mg}^{2+} / 5 \mathrm{~K}^{+}$aCSF incubation. On the other hand, a slice could show multiple (1-16) SLEs with or without developing SD. These results are consistent with the suppression, interference, or delay of neural activity after SD. For example, in rat slices in an interface chamber, SDs occur spontaneously after $0 \mathrm{Mg}^{2+} / 5 \mathrm{~K}^{+}$ aCSF exposure but only every $15 \mathrm{~min}$. For minutes after an $\mathrm{SD}$, responses to afferent stimulation in CA3 were weak (Scharfman, unpublished observations).

Both SD and SLEs developed in CA3 of mouse hippocampal slices in $0 \mathrm{Mg}^{2+} / 5 \mathrm{~K}^{+}$aCSF. When we examined the onset of $\mathrm{SD}$, we found trains of bursts that were very similar to SLEs, but shorter. Why these bursts are followed by SD sometimes, but otherwise they do not appear to do so, is not clear. It could be the bursts are triggered by different mechanisms, one due to entorhinal cortical input and one intrinsic to CA3. These different mechanisms may recruit feedforward or feedback inhibition more for SLEs and less for SD. Another possibility is that SLEs sometimes trigger SD and sometimes do not. This hypothesis is consistent with the idea that $\mathrm{SD}$ can be a natural anti-seizure mechanism where a seizure triggers $\mathrm{SD}$ and then $\mathrm{SD}$ suppresses activity for some time afterward (Tamim et al., 2021). 


\section{Propagation of Spreading Depolarization}

We found that SD typically began in CA3. We used two methods to identify this: electrical recording from more than one location and intrinsic optical imaging. Since our imaging field was limited, our method did not allow us to determine if SD began in an extrahippocampal area but use of a lower power objective could do so in the future. An increase of light transmittance propagated toward both CA1 and dentate gyrus with up to $30 \%$ transmittance change locally. Interestingly, SLEs only showed subtle changes in the intrinsic optical signals (2\%) but it was possible to visualize the small changes. It is possible that more detail might be clear with other wavelengths, such as $460-560 \mathrm{~nm}$ used previously (Mané and Müller, 2012). However, our recording at $775 \mathrm{~nm}$ was certainly sufficient.

Our analysis of SD propagation to the dentate gyrus is one of the few studies that have examined this issue. We found that SD propagated from CA3 to the dentate gyrus in a pattern that was unlike a unidirectional wave which approached the upper and lower blade simultaneously before the wavefront reached crest. Instead, SD circled around the dentate gyrus through upper blade, crest, and then lower blade. This pattern of SD spread from upper to lower blade was described before in response to $\mathrm{SD}$ elicited by focal $\mathrm{K}^{+}$stimulation to site near CA2 (Buchheim et al., 2002). Spread from upper to lower blade was also noted by Obeidat and Andrew (1998).

The reason for spread from upper to lower blade is not completely clear. There was no evidence that the lower blade had lower viability or better viability in our slices, based on viewing the cells in the slice with IR-DIC at $40 \times$. Patched GCs from the lower blade were healthy and similar to the GCs in the upper blade.

\section{Advantages and Disadvantages of Our Methods}

There are several advantages to the methods that we used. One advantage is to use the potent ability of reduced levels of $\left[\mathrm{Mg}^{2+}\right]_{0}$ to release its normal inhibition of SD (Santos et al., 2016). To preserve our slices during dissection and recovery, we used $\mathrm{Mg}^{2+}$ in the aCSF, but during recording and imaging it was removed. Use of this model is relevant to SD because there is a reduced $\mathrm{Mg}^{2+}$ block of the NMDA receptor, which is a receptor that becomes unblocked by depolarization as well as removal of $\mathrm{Mg}^{2+}$ from the aCSF. Depolarization occurs during the insult to the brain during TBI, seizures, and NMDA receptors are likely to become less blocked. Therefore, reducing $\left[\mathrm{Mg}^{2+}\right]_{\mathrm{o}}$ helps simulate the events during SD that occur during TBI and seizures. However, reducing $\left[\mathrm{Mg}^{2+}\right]_{0}$ also has other effects. As noted before, the dynamics of intracellular $\mathrm{Ca}^{2+}$ and frequency facilitation are affected, with facilitation promoted (Mody et al., 1987).

Regarding $\left[\mathrm{K}^{+}\right]_{\mathrm{o}}$, our model also was advantageous in that raising $\left[\mathrm{K}^{+}\right]_{\mathrm{o}}$ simulates the slow rise in $\left[\mathrm{K}^{+}\right]_{\mathrm{o}}$ after ischemic injury that leads to SD (Erdemli et al., 1998). In epilepsy, a rise in $\left[\mathrm{K}^{+}\right]_{\mathrm{o}}$ occurs at the onset of seizures and is thought to play a role in generation of the ictal state (Scharfman, 2015) which is typically followed by SD (Ssentongo et al., 2017). Therefore, by raising $\left[\mathrm{K}^{+}\right]_{\mathrm{o}}$ we simulated some of the changes that occur during $\mathrm{SD}$ in vivo in clinically relevant conditions.

On the other hand, as pointed out by Maslarova et al. (2011), in epileptic tissue there can be a resistance to $\mathrm{SD}$ and this was suggested to be due to a relatively high level of $\left[\mathrm{K}^{+}\right]_{\mathrm{o}}$ to foster $\mathrm{SD}$. The threshold concentration of $\left[\mathrm{K}^{+}\right]_{0}$ to facilitate SD in control slices was significantly lower than epileptic slices. Remarkably, the $\left[\mathrm{K}^{+}\right]_{\mathrm{o}}$ thresholds in that study were far above $10 \mathrm{mM}$ whereas in our tissue we raised $\left[\mathrm{K}^{+}\right]_{\mathrm{o}}$ from 3.5 to $5 \mathrm{mM}$, much less of a change. There were several differences in the studies that could explain the discrepancies in concentrations of $\left[\mathrm{K}^{+}\right]_{\mathrm{o}}$ required to promote SD, such as use of neocortex and human tissue, and other factors such as $500 \mu \mathrm{m}$-thick slices.

Use of $0 \mathrm{Mg}^{2+} / 5 \mathrm{~K}^{+}$aCSF is also useful as a comparison to osmotic stress or oxygen and glucose deprivation. A very comprehensive recent study compared osmotic stress, oxygen/glucose deprivation, and focal methods using normal aCSF (Frank et al., 2021). Both osmotic stress and oxygen/glucose deprivation led to spontaneous SD, and SD had incomplete recovery and injury compared to SD induced by focal methods in normal aCSF. There was greater spread of SD induced by osmotic stress and oxygen/glucose deprivation than the SD induced by focal methods in normal aCSF. In the present study, $0 \mathrm{Mg}^{2+} / 5 \mathrm{~K}^{+}$aCSF induced SD that appeared to recover completely and extensive neural injury did not seem to occur. One reason may be that there is less risk of injury with 0 $\mathrm{Mg}^{2+} / 5 \mathrm{~K}^{+}$aCSF because of the relative lack of osmotic stress and presence of oxygen and glucose. If that is true, the 0 $\mathrm{Mg}^{2+} / 5 \mathrm{~K}^{+}$aCSF provides a method to induce spontaneous $\mathrm{SD}$ that is not as severe in consequences, something that may be relevant to SD in migraine where neurons may not become damaged despite repetitive migraines. On the other hand, osmotic stress and oxygen/glucose deprivation may be more relevant to TBI and stroke that lead to severe damage.

Our slice model provides a method to expand the repertoire of a patch clamp rig to study spatiotemporal dynamics of both SD and SLEs without costly investments. While we found limitations with the classic submerged chamber, a slight modification to use a design more like an interface chamber circumvented the problem. This interface-like design can be fabricated or, as was the case in our study, purchased. The cost of a chamber was one of the few investments our methods required. Using a fast flow rate seemed important and is highly feasible.

Although rat slices could be used with the interface-like chamber, SD was not common. This might be circumvented by a mini-slice (if the problem is the large size of the slice, mentioned above), but then all subfields could not be imaged. Therefore, rat studies of SD are feasible with our methods but will require more animals to sample SD enough for statistical comparisons.

One advantage of our methods was that SD occurred faster than in prior studies using a classic interface chamber. SD only spontaneously developed after $2 \mathrm{~h}$ of $0 \mathrm{Mg}^{2+} / 5 \mathrm{~K}^{+}$aCSF exposure in a previous study and that spontaneous SD only was mentioned as occurring once (Mody et al., 1987). In comparison, we observed spontaneously developed SD within the first hour of $0 \mathrm{Mg}^{2+} / 5 \mathrm{~K}^{+}$aCSF incubation. 
The methods can potentially be used with ways to induce SD other than $0 \mathrm{Mg}^{2+} / 5 \mathrm{~K}^{+}$aCSF, such as local high $\mathrm{K}$ application, low calcium, ouabain, etc. It also can be used with transgenic mice, optogenetics, chemogenetics, and other methods currently used in research. Pharmacology is more readily used with the submerged chamber designs due to a smaller volume in the chamber compared to classic interface chambers. Therefore, drug studies and preclinical studies are facilitated by our methods.

The ability to induce both SD and SLEs is also useful for future studies of neuronal excitability and preclinical studies for epilepsy. For example, when a drug will be inhibitory to SD but facilitate SLEs is important to identify. The opposite, a drug that inhibits SLEs but leads to more SD is important.

Although we found no sex differences in the development of $\mathrm{SD}$ in mouse hippocampal slices, this may simply be due to the fact that the mice had not yet reached sexual maturity (Bell, 2018). Extending our findings into adulthood will be valuable in the future because of sex differences and hormonal regulation of in SD (Scharfman et al., 2003; Harte-Hargrove et al., 2013; Skucas et al., 2013; Scharfman and MacLusky, 2014). This topic has important translational implications because of sex differences in migraine and epilepsy (Scharfman and MacLusky, 2008, 2014; Vetvik and MacGregor, 2017).

There are also some disadvantages to the methods that we used. Although we were able to examine SD without a focal stimulus, the unpredictability of these SDs made it hard to trigger the start of video at the baseline before SD occurred. One method to circumvent the issue is to record throughout exposure to 0 $\mathrm{Mg}^{2+/} 5 \mathrm{~K}^{+}$aCSF, and as digital acquisition and storage improve such prolonged recording is likely to be easier.

\section{CONCLUSION}

Spreading depolarization and seizure-like events are both products of hypersynchronized activity of brain cells. We optimized a classic $0 \mathrm{Mg}^{2+} / 5 \mathrm{~K}^{+}$aCSF model for submerged hippocampal slices so both SD and SLEs are spontaneously developed within the first hour of $0 \mathrm{Mg}^{2+} / 5 \mathrm{~K}^{+}$aCSF exposure. We also extended our ability to record intrinsic optical signals without additional costs. With the relevance of SD and seizure activity to many pathological conditions and neurological disorders, this optimized model provides an opportunity to study $\mathrm{SD}$ with temporal and spatial advantages. It could also address

\section{REFERENCES}

Aitken, P. G., Breese, G. R., Dudek, F. F., Edwards, F., Espanol, M. T., Larkman, P. M., et al. (1995). Preparative methods for brain slices: a discussion. J. Neurosci. Methods 59, 139-149. doi: 10.1016/0165-0270(94)00 204-T

Anderson, T. R., and Andrew, R. D. (2002). Spreading depression: imaging and blockade in the rat neocortical brain slice. J. Neurophysiol. 88, 2713-2725. doi: 10.1152/jn.00321.2002

Anderson, W. W., Anderson, W. W., Lewis, D. V., Scott Swartzwelder, H., and Wilson, W. A. (1986). Magnesium-free medium activates seizure-like events in the rat hippocampal slice. Brain Res. 398, 215-219. doi: 10.1016/0006-8993(86) 91274-6 how SD and SLEs interact with each other in a controlled environment. We suggest that this model could be useful in testing mechanisms and validating treatment targets in preclinical studies.

\section{DATA AVAILABILITY STATEMENT}

The raw data supporting the conclusions of this article will be made available by the authors, without undue reservation.

\section{ETHICS STATEMENT}

The animal study was reviewed and approved by the Institutional Animal Care and Use Committee of The Nathan Kline Institute.

\section{AUTHOR CONTRIBUTIONS}

Y-LL and HES conceived and designed the study and wrote the manuscript. Y-LL performed the experiments and analyzed the data. Both authors contributed to the article and approved the submitted version.

\section{ACKNOWLEDGMENTS}

This study was supported by NIH grants AG-055328, MH109305, NS-037562, NS-081203, Pyramid Biosciences, and the NY State Office of Mental Health. We thank Karim Elayouby for the technical support in immunolabeling experiments. We are grateful to (by alphabetical order) Elissavet Chartampila, Kasey Gerencer, Swati Jain, John LaFrancois, and David AlcantaraGonzalez, Hannah Bernstein, Justin Botterill, Chiara Criscuolo, Áine Duffy, and Christos Lisgaras for their experimental supports and valuable discussions and feedback on the manuscript.

\section{SUPPLEMENTARY MATERIAL}

The Supplementary Material for this article can be found online at: https://www.frontiersin.org/articles/10.3389/fncel. 2021.761423/full\#supplementary-material

Avoli, M., D’Antuono, M., Louvel, J., Köhling, R., Biagini, G., Pumain, R., et al. (2002). Network and pharmacological mechanisms leading to epileptiform synchronization in the limbic system in vitro. Prog. Neurobiol. 68, 167-207. doi: 10.1016/S0301-0082(02)00077-1

Ayata, C., and Lauritzen, M. (2015). Spreading depression, spreading depolarizations, and the cerebral vasculature. Physiol. Rev. 95, 953-993. doi: 10.1152/physrev.00027.2014

Bell, M. R. (2018). Comparing postnatal development of gonadal hormones and associated social behaviors in rats, mice, and humans. Endocrinology 159, 2596-2613. doi: 10.1210/en.201800220

Billard, J. M., Jouvenceau, A., Lamour, Y., and Dutar, P. (1997). NMDA receptor activation in the aged rat: electrophysiological investigations in the CA1 area 
of the hippocampal slice ex vivo. Neurobiol. Aging 18, 535-542. doi: 10.1016/ S0197-4580(97)00104-8

Borck, C., and Jefferys, J. G. R. (1999). Seizure-like events in disinhibited ventral slices of adult rat hippocampus. J. Neurophysiol. 82, 2130-2142. doi: 10.1152/jn. 1999.82.5.2130

Botterill, J. J., Nogovitsyn, N., Caruncho, H. J., and Kalynchuk, L. E. (2017). Selective plasticity of hippocampal GABAergic interneuron populations following kindling of different brain regions. J. Comp. Neurol. 525, 389-406. doi: $10.1002 / \mathrm{cne} .24071$

Buchheim, K., Weissinger, F., Siegmund, H., Holtkamp, M., Schuchmann, S., and Meierkord, H. (2002). Intrinsic optical imaging reveals regionally different manifestation of spreading depression in hippocampal and entorhinal structures in vitro. Exp. Neurol. 175, 76-86. doi: 10.1006/exnr.2002.7893

Bugay, V., Bozdemir, E., Vigil, F. A., Chun, S. H., Holstein, D. M., Elliott, W. R., et al. (2020). A mouse model of repetitive blast traumatic brain injury reveals post-trauma seizures and increased neuronal excitability. J. Neurotrauma 37, 248-261. doi: 10.1089/neu.2018.6333

Case, L., and Broberger, C. (2013). A method for visually guided whole-cell recordings in brain slices exhibiting spontaneous rhythmic activity. J. Neurosci. Methods 212, 64-71. doi: 10.1016/j.jneumeth.2012.09.014

Churn, S. B., Anderson, W. W., and DeLorenzo, R. J. (1991). Exposure of hippocampal slices to magnesium-free medium produces epileptiform activity and simultaneously decreases calcium and calmodulin-dependent protein kinase II activity. Epilepsy Res. 9, 211-217. doi: 10.1016/0920-1211(91)90054-J

Croning, M. D., and Haddad, G. G. (1998). Comparison of brain slice chamber designs for investigations of oxygen deprivation in vitro. J. Neurosci. Methods 81, 103-111. doi: 10.1016/S0165-0270(98)00023-5

Czéh, G., Aitken, P. G., and Somjen, G. G. (1993). Membrane currents in CA1 pyramidal cells during spreading depression (SD) and SD-like hypoxic depolarization. Brain Res. 632, 195-208. doi: 10.1016/0006-8993(93)91154-K

D'Arcangelo, G., Tancredi, V., and Avoli, M. (2001). Intrinsic optical signals and electrographic seizures in the rat limbic system. Neurobiol. Dis. 8, 993-1005. doi: $10.1006 /$ nbdi.2001.0445

Dreier, J. P. (2011). The role of spreading depression, spreading depolarization and spreading ischemia in neurological disease. Nat. Med. 17, 439-447. doi: $10.1038 / \mathrm{nm} .2333$

Duffy, ÁM., Morales-Corraliza, J., Bermudez-Hernandez, K. M., Schaner, M. J., Magagna-Poveda, A., Mathews, P. M., et al. (2015). Entorhinal cortical defects in Tg2576 mice are present as early as 2-4 months of age. Neurobiol. Aging 36, 134-148. doi: 10.1016/j.neurobiolaging.2014.07.001

Edelstein, A., Amodaj, N., Hoover, K., Vale, R., and Stuurman, N. (2010). Computer control of microscopes using $\mu$ Manager. Curr. Protoc. Mol. Biol. 14, 1-17. doi: 10.1002/0471142727.mb1420s92

Edelstein, A. D., Tsuchida, M. A., Amodaj, N., Pinkard, H., Vale, R. D., and Stuurman, N. (2014). Advanced methods of microscope control using $\mu$ Manager software. J. Biol. Methods 1:e10. doi: 10.14440/jbm.2014.36

Eickhoff, M., Kovac, S., Shahabi, P., Ghadiri, M. K., Dreier, J. P., Stummer, W., et al. (2014). Spreading depression triggers ictaform activity in partially disinhibited neuronal tissues. Exp. Neurol. 253, 1-15. doi: 10.1016/j.expneurol.2013.12.008

Eikermann-Haerter, K., Negro, A., and Ayata, C. (2013). Spreading depression and the clinical correlates of migraine. Rev. Neurosci. 24, 353-363. doi: 10.1515/ revneuro-2013-0005

Erdemli, G., Xu, Y. Z., and Krnjević, K. (1998). Potassium conductance causing hyperpolarization of CA1 hippocampal neurons during hypoxia. J. Neurophysiol. 80, 2378-2390. doi: 10.1152/jn.1998.80.5.2378

Frank, R., Bari, F., Menyhárt, Á, and Farkas, E. (2021). Comparative analysis of spreading depolarizations in brain slices exposed to osmotic or metabolic stress. BMC Neurosci. 22:33. doi: 10.1186/s12868-021-00637-0

Gloveli, T., Albrecht, D., and Heinemann, U. (1995). Properties of low Mg2+ induced epileptiform activity in rat hippocampal and entorhinal cortex slices during adolescence. Dev. Brain Res. 87, 145-152. doi: 10.1016/0165-3806(95) 00069-P

Grube, P., Heuermann, C., Rozov, A., Both, M., Draguhn, A., and Hefter, D. (2021). Transient oxygen-glucose deprivation causes region- and cell type-dependent functional deficits in the mouse hippocampus in vitro. eNeuro 8:2021. doi: 10.1523/ENEURO.0221-21.2021
Haas, H. L., Schaerer, B., and Vosmansky, M. (1979). A simple perfusion chamber for the study of nervous tissue slices in vitro. J. Neurosci. Methods 1, 323-325. doi: 10.1016/0165-0270(79)90021-9

Hadley, A. (2010). CombineZP Image Stacking Software. Available online at: http: //micropics.org.uk/

Haglund, M. M., and Schwartzkroin, P. A. (1984). Seizure-like spreading depression in immature rabbit hippocampus in vitro. Brain Res. 316, 51-59.

Hájos, N., Ellender, T. J., Zemankovics, R., Mann, E. O., Exley, R., Cragg, S. J., et al. (2009). Maintaining network activity in submerged hippocampal slices: importance of oxygen supply. Eur. J. Neurosci. 29, 319-327. doi: 10.1111/j.14609568.2008.06577.x

Hájos, N., and Mody, I. (2009). Establishing a physiological environment for visualized in vitro brain slice recordings by increasing oxygen supply and modifying aCSF content. J. Neurosci. Methods 183, 107-113. doi: 10.1016/j. jneumeth.2009.06.005

Hamam, B. N., and Kennedy, T. E. (2003). Visualization of the dendritic arbor of neurons in intact $500 \mu \mathrm{m}$ thick brain slices. J. Neurosci. Methods 123, 61-67. doi: $10.1016 /$ S0165-0270(02)00341-2

Harte-Hargrove, L. C., Maclusky, N. J., and Scharfman, H. E. (2013). Brainderived neurotrophic factor-estrogen interactions in the hippocampal mossy fiber pathway: implications for normal brain function and disease. Neuroscience 239, 46-66. doi: 10.1016/j.neuroscience.2012.12.029

Hartings, J. A., Shuttleworth, C. W., Kirov, S. A., Ayata, C., Hinzman, J. M., Foreman, B., et al. (2017). The continuum of spreading depolarizations in acute cortical lesion development: examining Leão's legacy. J. Cereb. Blood Flow Metab. 37, 1571-1594. doi: 10.1177/0271678X16654495

Heinemann, U., Kann, O., and Schuchmann, S. (2006). "An overview of in vitro seizure models in acute and organotypic slices," in Models of Seizures and Epilepsy, eds A. Pitkänen, P. A. Schwartzkroin, and S. L. Moshé (Cambridge: Elsevier Academic Press), 35-44.

Juzekaeva, E., Gainutdinov, A., Mukhtarov, M., and Khazipov, R. (2020). Reappraisal of anoxic spreading depolarization as a terminal event during oxygen-glucose deprivation in brain slices in vitro. Sci. Rep. 10:18970. doi: 10.1038/s41598-020-75975-w

Kim, Y. K., Yang, E. J., Cho, K., Lim, J. Y., and Paik, N. J. (2014). Functional recovery after ischemic stroke is associated with reduced gabaergic inhibition in the cerebral cortex: a GABA PET study. Neurorehabil. Neural Repair 28, 576-583. doi: 10.1177/1545968313520411

Kojima, H., Kowada, M., and Bragdon, A. C. (1991). Mechanism of electrographic seizure generation in the rat brain slice in low magnesium medium: modulatory effect of interictal bursting on seizure generation. Jpn. J. Psychiatry Neurol. 45, 279-283. doi: 10.1111/j.1440-1819.1991.tb02472.x

Kovács, R., Gutiérrez, R., Kivi, A., Schuchmann, S., Gabriel, S., and Heinemann, U. (1999). Acute cell damage after low Mg2+-induced epileptiform activity in organotypic hippocampal slice cultures. Neuroreport 10, 207-213. doi: 10.1097/ 00001756-199902050-00002

Leão, A. A. P. (1944). Spreading depression of activity in the cerebral cortex. J. Neurophysiol. 7, 359-390. doi: 10.1152/jn.1944.7.6.359

Lewis, D. V., Jones, L. S., and Mott, D. D. (1990). Hippocampal epileptiform activity induced by magnesium-free medium: differences between areas CA1 and CA2-3. Epilepsy Res. 6, 95-101. doi: 10.1016/0920-1211(90)90083-8

Lewis, D. V., Jones, L. S., and Swartzwelder, H. S. (1989). The effects of baclofen and pertussis toxin on epileptiform activity induced in the hippocampal slice by magnesium depletion. Epilepsy Res. 4, 109-118. doi: 10.1016/0920-1211(89) 90015-6

Lian, X. Y., and Stringer, J. L. (2004). Astrocytes contribute to regulation of extracellular calcium and potassium in the rat cerebral cortex during spreading depression. Brain Res. 1012, 177-184. doi: 10.1016/j.brainres.2004. 04.011

Lindquist, B. E., and Shuttleworth, C. W. (2012). Adenosine receptor activation is responsible for prolonged depression of synaptic transmission after spreading depolarization in brain slices. Neuroscience 223, 365-376. doi: 10.1016/j. neuroscience.2012.07.053

Lorente De Nó, R. (1934). Studies on the structure of the cerebral cortex II. Continuation of the study of the ammonic system. J. Psychol. Neurol. 46, 113-177. 
MacVicar, B. A., and Hochman, D. (1991). Imaging of synaptically evoked intrinsic optical signals in hippocampal slices. J. Neurosci. 11, 1458-1469. doi: 10.1523/ jneurosci.11-05-01458.1991

Mané, M., and Müller, M. (2012). Temporo-Spectral imaging of intrinsic optical signals during hypoxia-induced spreading depression-like depolarization. PLoS One 7:e43981. doi: 10.1371/journal.pone.0043981

Martens-Mantai, T., Speckmann, E. J., and Gorji, A. (2014). Propagation of cortical spreading depression into the hippocampus: the role of the entorhinal cortex. Synapse 68, 574-584. doi: 10.1002/syn.21769

Maslarova, A., Alam, M., Reiffurth, C., Lapilover, E., Gorji, A., and Dreier, J. P. (2011). Chronically epileptic human and rat neocortex display a similar resistance against spreading depolarization in vitro. Stroke 42, 2917-2922. doi: 10.1161/STROKEAHA.111.621581

Mody, I., Lambert, J. D., and Heinemann, U. (1987). Low extracellular magnesium induces epileptiform activity and spreading depression in rat hippocampal slices. J. Neurophysiol. 57, 869-888. doi: 10.1152/jn.1987.57.3.869

Morris, G., Jiruska, P., Jefferys, J. G. R., and Powell, A. D. (2016). A new approach of modified submerged patch clamp recording reveals interneuronal dynamics during epileptiform oscillations. Front. Neurosci. 10:519. doi: 10.3389/fnins. 2016.00519

Neuman, R. S., Cherubini, E., and Ben-Ari, Y. (1989). Endogenous and network bursts induced by $\mathrm{N}$-methyl-D-aspartate and magnesium free medium in the CA3 region of the hippocampal slice. Neuroscience 28, 393-399. doi: 10.1016/ 0306-4522(89)90186-3

Noseda, R., and Burstein, R. (2013). Migraine pathophysiology: anatomy of the trigeminovascular pathway and associated neurological symptoms, cortical spreading depression, sensitization, and modulation of pain. Pain 154, S44-S53. doi: 10.1016/j.pain.2013.07.021

Obeidat, A. S., and Andrew, R. D. (1998). Spreading depression determines acute cellular damage in the hippocampal slice during oxygen/glucose deprivation. Eur. J. Neurosci. 10, 3451-3461. doi: 10.1046/j.1460-9568.1998.00358.x

Obeidat, A. S., Jarvis, C. R., and Andrew, R. D. (2000). Glutamate does not mediate acute neuronal damage after spreading depression induced by $\mathrm{O} 2 /$ glucose deprivation in the hippocampal slice. J. Cereb. Blood Flow Metab. 20, 412-422. doi: 10.1097/00004647-200002000-00024

Reyes-Garcia, S. Z., Scorza, C. A., Araújo, N. S., Ortiz-Villatoro, N. N., Jardim, A. P., Centeno, R., et al. (2018). Different patterns of epileptiform-like activity are generated in the sclerotic hippocampus from patients with drugresistant temporal lobe epilepsy. Sci. Rep. 8:7116. doi: 10.1038/s41598-01825378-9

Rogawski, M. A. (2013). "Migraine and epilepsy-shared mechanisms within the family of episodic disorders," in Jasper's Basic Mechanisms of the Epilepsies, eds J. L. Noebels, M. Avoli, M. A. Rogawski, R. W. Olsen, and A. V. Delgado-Escueta (Bethesda: National Center for Biotechnology Information), 930-944.

Sanchez-Vives, M. V., and McCormick, D. A. (2000). Cellular and network mechanisms of rhytmic recurrent activity in neocortex. Nat. Neurosci. 3, 10271034. doi: 10.1038/79848

Santos, E., León, F., Silos, H., Sanchez-Porras, R., Shuttleworth, C. W., Unterberg, A., et al. (2016). Incidence, hemodynamic, and electrical characteristics of spreading depolarization in a swine model are affected by local but not by intravenous application of magnesium. J. Cereb. Blood Flow Metab. 36, 20512057. doi: 10.1177/0271678X16671317

Scharfman, H. E. (1993a). Characteristics of spontaneous and evoked EPSPs recorded from dentate spiny hilar cells in rat hippocampal slices. J. Neurophysiol. 70, 742-757. doi: 10.1152/jn.1993.70.2.742

Scharfman, H. E. (1993b). Spiny neurons of area CA3c in rat hippocampal slices have similar electrophysiological characteristics and synaptic responses despite morphological variation. Hippocampus 3, 9-28. doi: 10.1002/hipo.450030103

Scharfman, H. E. (1995). Electrophysiological evidence that dentate hilar mossy cells are excitatory and innervate both granule cells and interneurons. J. Neurophysiol. 74, 179-194. doi: 10.1152/jn.1995.74.1.179

Scharfman, H. E. (1997). Hyperexcitability in combined entorhinal/hippocampal slices of adult rat after exposure to brain-derived neurotrophic factor. J. Neurophysiol. 78, 1082-1095. doi: 10.1152/jn.1997.78.2.1082

Scharfman, H. E. (2007). The CA3 "backprojection" to the dentate gyrus. Prog Brain Res. 63, 627-637. doi: 10.1016/S0079-6123(07)63034-9

Scharfman, H. E. (2015). "Epilepsy," in Neurobiology of Brain Disorders: Biological Basis of Neurological and Psychiatric Disorders, eds M. J.
Zigmond, L. P. Rowland, and J. T. Coyle (Amsterdam: Elsevier Inc.), 236-261.

Scharfman, H. E., and MacLusky, N. J. (2008). Estrogen-growth factor interactions and their contributions to neurological disorders. Headache 48, S77-S89. doi: 10.1111/j.1526-4610.2008.01200.x

Scharfman, H. E., and MacLusky, N. J. (2014). Sex differences in the neurobiology of epilepsy: a preclinical perspective. Neurobiol. Dis. 72, 180-192. doi: 10.1016/ j.nbd.2014.07.004

Scharfman, H. E., Mercurio, T. C., Goodman, J. H., Wilson, M. A., and MacLusky, N. J. (2003). Hippocampal excitability increases during the estrous cycle in the rat: a potential role for brain-derived neurotrophic factor. J. Neurosci. 23, 11641-11652.

Scharfman, H. E., Smith, K. L., Goodman, J. H., and Sollas, A. L. (2001). Survival of dentate hilar mossy cells after pilocarpine-induced seizures and their synchronized burst discharges with area CA3 pyramidal cells. Neuroscience 104, 741-759. doi: 10.1016/S0306-4522(01)00132-4

Schneider, C. A., Rasband, W. S., and Eliceiri, K. W. (2012). NIH Image to ImageJ: 25 years of image analysis. Nat. Methods 9, 671-675. doi: 10.1038/nmeth.2089

Schindelin, J., Arganda-Carreras, I., Frise, E., Kaynig, V., Longair, M., Pietzsch, T., et al. (2012). Fiji: an open-source platform for biological-image analysis. Nat. Methods 9, 676-82. doi: 10.1038/nmeth.2019

Schuchmann, S., Meierkord, H., Stenkamp, K., Breustedt, J., Windmüller, O., Heinemann, U., et al. (2002). Synaptic and nonsynaptic ictogenesis occurs at different temperatures in submerged and interface rat brain slices. J. Neurophysiol. 87, 2929-2935. doi: 10.1152/jn.2002.87.6.2929

Schwartzkroin, P. A. (1975). Characteristics of CA1 neurons recorded intracellularly in the hippocampal in vitro slice preparation. Brain Res. 85, 423-436. doi: 10.1016/0006-8993(75)90817-3

Skucas, V. A., Duffy, ÁM., Harte-Hargrove, L. C., Magagna-Poveda, A., Radman, T., Chakraborty, G., et al. (2013). Testosterone depletion in adult male rats increases mossy fiber transmission, LTP, and sprouting in area CA3 of Hippocampus. J. Neurosci. 33, 2338-2355. doi: 10.1523/jneurosci.3857-12.2013

Sloviter, R. S. (1991). Permanently altered hippocampal structure, excitability, and inhibition after experimental status epilepticus in the rat: the "dormant basket cell" hypothesis and its possible relevance to temporal lobe epilepsy. Hippocampus 1, 41-66. doi: 10.1002/hipo.450010106

Soltesz, I., Smetters, D. K., and Mody I. (1995). Tonic inhibition originates from synapses close to the soma. Neuron 14, 1273-1283. doi: 10.1016/0896-6273(95) 90274-0

Snow, R. W., Taylor, C. P., and Dudek, F. E. (1983). Electrophysiological and optical changes in slices of rat hippocampus during spreading depression. J. Neurophysiol. 50, 561-572. doi: 10.1152/jn.1983.50. 3.561

Somjen, G. G. (2001). Mechanisms of spreading depression and hypoxic spreading depression-like depolarization. Physiol. Rev. 81, 1065-1096. doi: 10.1152/ physrev.2001.81.3.1065

Ssentongo, P., Robuccio, A. E., Thuku, G., Sim, D. G., Nabi, A., Bahari, F., et al. (2017). A Murine Model to Study Epilepsy and SUDEP Induced by Malaria Infection. Sci. Rep. 7:43652. doi: 10.1038/srep43652

Steffensen, A. B., Sword, J., Croom, D., Kirov, S. A., and MacAulay, N. (2015). Chloride cotransporters as a molecular mechanism underlying spreading depolarization-induced dendritic beading. J. Neurosci. 35, 12172-12187. doi: 10.1523/JNEUROSCI.0400-15.2015

Tamim, I., Chung, D. Y., de Morais, A. L., Loonen, I. C. M., Qin, T., Misra, A., et al. (2021). Spreading depression as an innate antiseizure mechanism. Nat. Commun. 12:2206. doi: 10.1038/s41467-021-22464-x

Umorin, M. (2002). Stack Focuser. Available online at: https://imagej.nih.gov/ij/ plugins/stack-focuser.html.

Varga, D. P., Menyhárt, Á, Pósfai, B., Császár, E., Lénárt, N., Cserép, C., et al. (2020). Microglia alter the threshold of spreading depolarization and related potassium uptake in the mouse brain. J. Cereb. Blood Flow Metab. 40, S67-S80. doi: 10.1177/0271678X19900097

Vetvik, K. G., and MacGregor, E. A. (2017). Sex differences in the epidemiology, clinical features, and pathophysiology of migraine. Lancet Neurol. 16, 76-87. doi: 10.1016/S1474-4422(16)30293-9

von Krosigk, M., Bal, T., and McCormick, D. A. (1993). Cellular mechanisms of a synchronized oscillation in the thalamus. Science 261, 361-364. doi: 10.1126/ science. 8392750 
Wahab, A., Heinemann, U., and Albus, K. (2009). Effects of $\gamma$-aminobutyric acid (GABA) agonists and a GABA uptake inhibitor on pharmacoresistant seizure like events in organotypic hippocampal slice cultures. Epilepsy Res. 86, 113-123. doi: 10.1016/j.eplepsyres.2009.05.008

Witter, M. P., Doan, T. P., Jacobsen, B., Nilssen, E. S., and Ohara, S. (2017). Architecture of the entorhinal cortex a review of entorhinal anatomy in rodents with some comparative notes. Front. Syst. Neurosci. 11:46. doi: 10.3389/fnsys. 2017.00046

Conflict of Interest: The authors declare that the research was conducted in the absence of any commercial or financial relationships that could be construed as a potential conflict of interest.
Publisher's Note: All claims expressed in this article are solely those of the authors and do not necessarily represent those of their affiliated organizations, or those of the publisher, the editors and the reviewers. Any product that may be evaluated in this article, or claim that may be made by its manufacturer, is not guaranteed or endorsed by the publisher.

Copyright (c) $2021 \mathrm{Lu}$ and Scharfman. This is an open-access article distributed under the terms of the Creative Commons Attribution License (CC BY). The use, distribution or reproduction in other forums is permitted, provided the original author(s) and the copyright owner(s) are credited and that the original publication in this journal is cited, in accordance with accepted academic practice. No use, distribution or reproduction is permitted which does not comply with these terms. 